\title{
Temperature distribution in a Newtonian fluid injected between two semi-infinite plates.
}

\section{H.J.J. Gramberg and A.A.F. van de Ven}

Eindhoven University of Technology, Mathematics and Computing Science, P.O.Box 513, 5600 MB Eindhoven, The Netherlands

\begin{abstract}
The analysis of the temperature distribution in time and place of a hot heatconducting Newtonian fluid injected between two cooled parallel plates is presented. The 2-dimensional flow has a free flow front moving with constant velocity. The kernel of the fluid remains almost at the inlet temperature, but at the walls boundary layers occur with steeply descending temperature. The inner solutions inside these boundary layers are determined. To this end, the total region is divided into three distinct regions: the region $G_{I}$ far behind the flow front, the flow front region $G_{I I}$, and the intermediate region $G_{I I I}$ between $G_{I}$ and $G_{I I}$. The asymptotics owing to each region are presented. The fundamental small parameter here is the thickness-to-length ratio of the 2-dimensional flow region. In most of the cases, similarity solutions are found. In the flow front region, for the formulation of the inner solution a Wiener-Hopf technique is used. Via matching procedures, the separate boundary layers are linked to each other to form one global boundary layer for the whole front region. All calculations in this paper are performed by analytical means, and all results are in analytical form. Comparison of our results with numerical solutions shows perfect agreement.
\end{abstract}

Keywords: injection moulding; free flow front; temperature distribution; boundary layers.

\section{Introduction}

During the filling phase of an injection moulding process several peculiar phenomena occur, especially close to the flow front, which is a free boundary for the flow. Hence, the shape of the flow front is an unknown of the flow problem. At the flow front, the flow is a so-called fountain flow. For the 2-dimensional problem of an injection flow between two parallel plates, the mould walls, and for a Newtonian fluid, this flow problem is solved completely by analytical means in [1]. In that paper, no thermal effects are considered. In the present paper, we shall use the results of [1] to calculate, again as much as possible by analytical means, the temperature distribution in the injected fluid, taking into account the effects inherent to the flow front and the fountain flow there, according to [1]. We assume that the fluid is injected at a high temperature, whereas the parallel mould walls are kept at a uniform and constant lower temperature. So, the mould walls will cool down the hot fluid, thus causing temperature gradients in the fluid, that are most strong near those walls. An analogous flow 
process occurs for instance in the injection moulding of compact discs. In this kind of processes, thermal effects reveal themselves in, amongst others, influences on the viscosity which is strongly temperature dependent. The effects due to temperature-dependent viscosities on the injection moulding of polymers melts have been investigated by Mavridis et al. [2], Wall and Wilson [3], Attia [4], Pinarbasy and Ozalp [5], Estep et al. [6], Becker and McKinley [7], and Nguyen-Chung and Mennig [8]. In Mavridis et al. [2], a temperature profile at the end of the filling phase and far behind the flow front has been calculated; see their Fig. 7. Their result shows that the temperature in the kernel of the flow (approximately $2 / 3$ of the width of the gap between the plates) is still equal to the hot inlet temperature, whereas the fluid cools down to the wall temperature in two thin layers near the walls. Comparable results can be found in [7, Fig. 6]. Also in the other references temperature profiles are calculated, but this is always done by numerical means, in which the influence of (the fountain flow at) the flow front is not taken into account (this in contrast to the present paper). Only in [8], it looks as if the development of the flow front is taken into account. However, nowhere in this paper boundary conditions for the temperature on the flow front are specified. Moreover since no results of the temperature simulations are presented, a comparison with our results is impossible. In some of the mentioned papers, [3], [5], and [6], the calculated temperature distribution and, more specifically, its influence on the temperature-dependent viscosity, are used to investigate the (in)stability of the channel flow; significant influences on the stability of the flow are found. One aspect of the stability behaviour is the so-called flow front instability: in experiments on injection between parallel plates, it is observed that the motion of the flow front becomes instationary and asymmetric; the front starts wobbling, [9] or [10, Sect. 10.3]. It is believed that this front instability is caused by either thermal or elastic effects, or by a combination of the two.

To get some insight into the mechanism, we study a simplified problem in which the polymer is considered to be an incompressible Newtonian fluid, where the viscosity $\eta$ depends on the temperature $T$ only. The total problem is split up into a temperature problem and a velocity problem, which are coupled. In case the flow front is symmetric and stationary, moving with constant speed, the shape of the flow front, the streamlines, and the complete velocity field are calculated in [1]. When the flow front is slightly perturbed to an asymmetric shape and the temperature dependence of the viscosity is taken into account, the evolution in time of the flow front can be analysed, leading to a statement about the (in)stability of the flow front motion; this is explained in somewhat more detail in [9] or [10], and will be the subject of a forthcoming thesis of Gramberg [11].

In this paper, we will look at the pure temperature problem, in which we calculate $T$ near and behind the flow front. This leads to a convection-diffusion problem, in the convective term of which the velocity $\mathbf{v}$ is used as found in [1]. The problem is formulated for a 2-dimensional region between two parallel plates, the mould. The mould is partially filled with polymer melt. At the inlet, the hot fluid is injected. As the walls are cooled, the melt gets also cooled at the walls. Due to the short process time which we consider here (only the filling phase is considered), the kernel of the injected fluid does not cool much and, therefore, will remain (almost) at the inlet temperature. Consequently, there will occur boundary layers at the walls, in which the temperature is lower than the inlet temperature. In these narrow layers asymptotics will be used to formulate and solve the temperature problem. It turns out that the asymptotics in a layer very close to the flow front differ essentially from those in a layer far behind the flow front. For both cases the asymptotic or boundary layer problems will be solved here. The fluid model we consider here is simple: a Newtonian fluid with constant 
viscosity, and in the heat equation the viscous dissipation term is neglected.

In Section 2, the mathematical formulation of the temperature problem is presented. Three different regions are introduced there:

1. The region far behind the flow front $\left(G_{I}\right)$.

2. The flow front region $\left(G_{I I}\right)$.

3. The intermediate region $\left(G_{I I I}\right)$ between $G_{I}$ and $G_{I I}$.

In each of these regions different asymptotics apply. However the fundamental parameter is everywhere the thickness-to-length ratio of the 2-dimensional flow region. In Sections 3, 4, and 5, successively the asymptotic solutions in the boundary layers in $G_{I}, G_{I I}$ and $G_{I I I}$ are derived. In Sections 3 and 5, the solution is found via the introduction of a similarity solution, whereas in Section 4, a solution is obtained via a Wiener-Hopf technique. In Section 5, the different boundary layers are linked to each other by a matching procedure. A review of the main results and the conclusions is given in Section 6 .

\section{Mathematical formulation}

The system we consider here consists of two semi-infinite parallel plates at a distance $2 h$, occupying the two half-planes $\{(X, Y, Z) \mid X>0 \wedge Y= \pm h\}$. Here, we have introduced a system of Cartesian coordinates, $X, Y$ and $Z$, where $Y$ is the coordinate in the direction perpendicular to the plates; see Figure 1. Through the cross-section $\{X=0 \wedge-h<Y<h\}$ a fluid is injected and it starts to flow between the plates with a velocity $\mathbf{V}$. We consider the problem to be two-dimensional, meaning that $\mathbf{V}$ is independent of $Z$, and that the $Z$ component of $\mathbf{V}$ is zero: $\mathbf{V}=U(X, Y, t) \mathbf{e}_{X}+V(X, Y, t) \mathbf{e}_{Y}$.

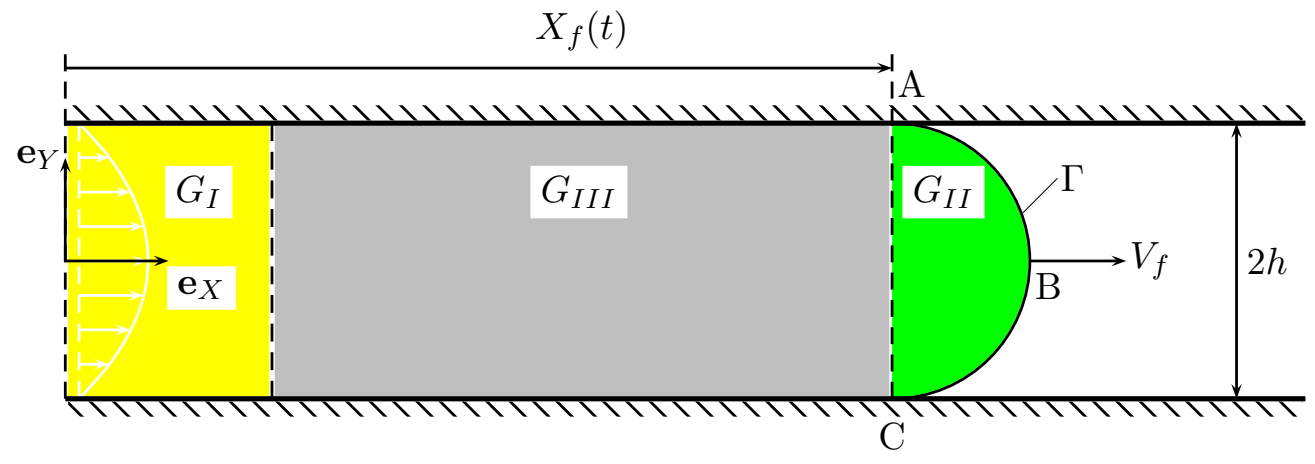

Figure 1: The flow region with moving front between two plates, divided into three regions: the region far behind the flow front $G_{I}$, the flow front region $G_{I I}$, and the intermediate region $G_{I I I}$.

The region between the plates that at a certain time $t$ is filled with fluid, is denoted by $G$ and is bounded by the walls at $Y= \pm h$, the inlet $X=0$, and the flow front, curve $A B C=\Gamma$ 
as indicated in Figure 1. The region $G$ is given by

$$
G=\left\{(X, Y) \mid-h<Y<h \wedge 0<X<X_{f}(t)+\xi_{f}(Y, t)\right\},
$$

and the flow front $\Gamma(\equiv A B C)$ by

$$
\Gamma=\left\{(X, Y) \mid X=X_{f}(t)+\xi_{f}(Y, t) \wedge-h<Y<h\right\},
$$

where $X_{f}(t)$ is the $X$-position of point $A$ (or $C$ ), and $\xi_{f}(Y, t)$ is the horizontal position with respect to the line $A C$ and at time $t$ of a point on the flow front $\Gamma$ having vertical position $Y$. The normal on $\Gamma$ in a certain point $(X, Y)=\left(X_{f}(t)+\xi_{f}(Y, t), Y\right)$ and at time $t$ is given by

$$
\mathbf{n}=\mathbf{n}(Y, t)=\frac{1}{\sqrt{1+\left(\xi_{f, Y}(Y, t)\right)^{2}}}\left(1,-\xi_{f, Y}(Y, t)\right),
$$

where (.), $Y$ denotes differentiation with respect to $Y$.

For the calculations of the temperature in $G$, to be presented in this paper, it is convenient to divide $G$ into three disjoint regions; see Figure 1 . Here, region $G_{I}$ is the region far behind the flow front, region $G_{I I}$ is the flow front region, and region $G_{I I I}$ is the transition region between $G_{I}$ and $G_{I I}$ (so, $G=G_{I} \cup G_{I I} \cup G_{I I I}$ ). In this paper we will calculate the temperature in the regions $G_{I}, G_{I I}, G_{I I I}$, separately.

Here, we assume that the flow front has a fixed shape moving with constant speed $V_{f}$ in the $X$-direction, and that it is symmetric with respect to $Y$. This means that $X_{f}(t)=V_{f} t$ and $\xi_{f}=\xi_{f}(Y)=\xi_{f}(-Y)$.

For an incompressible Newtonian fluid, the velocity near the flow front can be calculated by analytical means; see [1], where it was found that the shape of the flow front looks very much like a semi-circle. We introduce a comoving frame $\{o x y\}$ with the dimensionless coordinates

$$
x=\frac{X-V_{f} t}{h}, \quad y=\frac{Y}{h} .
$$

In this co-moving frame, the flow front is given by (in dimensionless form)

$$
x=x_{f}(y)=\frac{1}{h} \xi_{f}\left(\frac{Y}{h}\right), \quad-1<y<1 .
$$

Since the shape of the flow front resembles a semi-circle, the characteristic measure of length in the $X$-direction in $G_{I I}$ is of the same order as the characteristic length $h$ in the $Y$-direction, meaning that both $x$ and $y$ are $O(1)$ in $G_{I I}$.

In the flow front region, the velocity with respect to the flow front is independent of time $t$, meaning that the components of the velocity $\mathbf{V}=U \mathbf{e}_{x}+V \mathbf{e}_{y}$ may be written there as

$$
U(X, Y, t)=V_{f}(1+u(x, y)), \quad V(X, Y, t)=V_{f} v(x, y),
$$

where $\mathbf{v}=u(x, y) \mathbf{e}_{x}+v(x, y) \mathbf{e}_{y}$ is the dimensionless velocity with respect to the co-moving frame.

In the sequel, we consider the problem for larger times, such that $X_{f} t \gg h$. This implies that far behind the flow front the flow is considered to be fully developed and independent of time. Accordingly the velocity far behind the flow front is given by

$$
U=U_{P}(Y):=\frac{3}{4} V_{f}\left(1-\left(\frac{Y}{h}\right)^{2}\right), \quad V=0 .
$$


We assume that the temperature $T(X, Y, t)$ in $G$ is governed by the energy equation; see [12, eq. (4.64)]. To simplify the problem, we neglect the effects due to energy dissipation and thermal expansion, and we assume $\rho$, the density, $c_{p}$, the specific heat, and $\lambda$ the thermal conductivity, to be constant, rendering the diffusion-convection equation for the temperature:

$$
\rho c_{p}\left(\frac{\partial T}{\partial t}+U \frac{\partial T}{\partial X}+V \frac{\partial T}{\partial Y}\right)=\lambda\left(\frac{\partial^{2} T}{\partial X^{2}}+\frac{\partial^{2} T}{\partial Y^{2}}\right) .
$$

This differential equation has to be supplemented with a set of boundary conditions, such as:

- The temperature at the inlet $X=0$ of the mould is assumed to be constant and equal to $T_{i}$.

- The temperature of the fluid at the walls of the mould is taken to be constant and equal to $T_{w}\left(<T_{i}\right)$. We assume that the temperature of the fluid in contact with the walls is also equal to $T_{w}$; hence, $T(X, Y)=T_{w}$, for $Y= \pm h$ and $X<V_{f} t$.

- The thermal conductivity of air is much smaller than the thermal conductivity of the fluid (e.g. a polymer). Therefore, we assume that the flow front may be taken to be thermally insulated, meaning that for $(X, Y) \in \Gamma, \partial T / \partial n=0$.

Since the types of flow are quite different in $G_{I}$ and in $G_{I I}$, the respective convective terms in (8) are also quite different. Therefore, we have to distinguish between the behaviour of the temperature far behind the flow front (in $G_{I}$ ), and the behaviour near the flow front (in $G_{I I}$ ):

- Behaviour of $T$ in $G_{I}$, far behind the flow front.

In $G_{I}$, we assume the effect of the flow front negligible and consequently the temperature may be assumed stationary, yielding $T=T_{I}(X, Y)$, for $(X, Y) \in G_{I}$. Here, $T_{I}(X, Y)$ is governed by the equation (derived from (8) with use of (7))

$$
\frac{3}{4} \rho c_{p} V_{f}\left(1-\left(\frac{Y}{h}\right)^{2}\right) \frac{\partial T_{I}}{\partial X}=\lambda \Delta T_{I}(X, Y) .
$$

In $G_{I}$, the following two boundary conditions for $T_{I}$ are relevant (and as our further analysis will show, also sufficient):

$$
\begin{aligned}
& -T_{I}(0, Y)=T_{i}, \quad \text { for } \quad-h<Y<h ; \\
& -T_{I}(X, \pm h)=T_{w}, \quad \text { for } \quad(X, \pm h) \in G_{I} .
\end{aligned}
$$

- Behaviour of $T$ in $G_{I I}$, the flow front region.

In $G_{I I}$, the temperature is assumed to be stationary with respect to the co-moving frame $\{o x y\}$, yielding $T(X, Y, t)=T_{I I}(x, y)$. Transforming $(8)$ to the $(x, y)$-coordinate system, with use of $(6)$, we obtain

$$
h V_{f} \rho c_{p}\left(u \frac{\partial T_{I I}}{\partial x}+v \frac{\partial T_{I I}}{\partial y}\right)=\lambda\left(\frac{\partial^{2} T_{I I}}{\partial x^{2}}+\frac{\partial^{2} T_{I I}}{\partial y^{2}}\right) .
$$

The boundary conditions for $T_{I I}$ are 
- At the flow front $x=x_{f}(y)$, the normal derivative of $T_{I I}$ is equal to zero, so

$$
\begin{aligned}
& \frac{\partial T_{I I}}{\partial x}-x_{f}^{\prime}(y) \frac{\partial T_{I I}}{\partial y}=0, \quad \text { on } \Gamma ; \\
& -T_{I I}(x, \pm 1)=T_{w}, \quad \text { for } x \geq 0 .
\end{aligned}
$$

- Behaviour of $T$ in $G_{I I I}$, the transition region between $G_{I}$ and $G_{I I}$.

This region is needed to match the solution for $T$ in $G_{I}$ to that in $G_{I I}$. How this will be done, will be specified further on.

We proceed by making the equations derived thus far dimensionless. We have defined $x$, $y$ and $\mathbf{v}$ such that they are already dimensionless and of order $O(1)$ in $G_{I I}$. We introduce dimensionless variables $\hat{X}, \hat{Y}, \hat{\mathbf{V}}, \hat{T}_{I}$ and $\hat{T}_{I I}$, by

$$
\hat{X}=\frac{X}{R}, \quad \hat{Y}=\frac{Y}{h}, \quad \hat{\mathbf{V}}=\frac{4}{3 V_{f}} \mathbf{V}, \quad \hat{T}=\frac{T-T_{w}}{T_{i}-T_{w}} .
$$

Here, $R$ is a characteristic length in the $X$-direction of region $G_{I}$. Since we are looking at the behaviour for larger times, and far behind the flow front, we assume that $R / h \gg 1$ (see also Table 1).

Substitution of these scalings into the differential equations and boundary conditions for $T_{I}$ and $T_{I I}$ as derived previously, renders the following set of dimensionless equations for $\hat{T}_{I}$ and $\hat{T}_{I I}:$

- The dimensionless temperature $\hat{T}_{I}=\hat{T}_{I}(\hat{X}, \hat{Y})$ satisfies the differential equation

$$
\left(1-\hat{Y}^{2}\right) \frac{\partial \hat{T}_{I}}{\partial \hat{X}}=\lambda_{I}\left(\delta^{2} \frac{\partial^{2} \hat{T}_{I}}{\partial \hat{X}^{2}}+\frac{\partial^{2} \hat{T}_{I}}{\partial \hat{Y}^{2}}\right),
$$

where the dimensionless group $\lambda_{I}$ is given by

$$
\lambda_{I}=\frac{4 R \lambda}{3 \rho c_{p} h^{2} V_{f}},
$$

and $\delta=h / R$, with $0<\delta \ll 1$. Therefore, we will neglect the term preceded by $\delta^{2}$ in the diffusive term of (13)), which leads us to

$$
\left(1-\hat{Y}^{2}\right) \frac{\partial \hat{T}_{I}}{\partial \hat{X}}=\lambda_{I} \frac{\partial^{2} \hat{T}_{I}}{\partial \hat{Y}^{2}}
$$

The boundary conditions for $T_{I}$ transform to

$$
\begin{aligned}
& \text { - } \hat{T}_{I}(0, \hat{Y})=1, \quad \text { for } \quad-1<\hat{Y}<1 \text {; } \\
& \text { - } \hat{T}_{I}(\hat{X}, \pm 1)=0, \quad \text { for } \quad \hat{X}>0 .
\end{aligned}
$$

- The differential equation for the dimensionless temperature $\hat{T}_{I I}=\hat{T}_{I I}(x, y)$ is

$$
u \frac{\partial \hat{T}_{I I}}{\partial x}+v \frac{\partial \hat{T}_{I I}}{\partial y}=\lambda_{I I}\left(\frac{\partial^{2} \hat{T}_{I I}}{\partial x^{2}}+\frac{\partial^{2} \hat{T}_{I I}}{\partial y^{2}}\right),
$$


where $\lambda_{I I}$ is given by

$$
\lambda_{I I}=\delta \lambda_{I}=\frac{\lambda}{\rho c_{p} h V_{f}} .
$$

The boundary conditions for $\hat{T}_{I I}$ are

- At $x=x_{f}(y)$, and for $-1 \leq y \leq 1$ :

$$
\frac{\partial \hat{T}_{I I}}{\partial x}=x_{f}^{\prime}(y) \frac{\partial \hat{T}_{I I}}{\partial y} ;
$$

- for $x \leq 0$,

$$
\hat{T}_{I I}(x, \pm 1)=0 .
$$

Table 1: Numerical values for material coefficients

\begin{tabular}{|l|c|l|}
\hline symbol & order of magnitude & unit \\
\hline$R$ & $6 \cdot 10^{-2}$ & $\mathrm{~m}$ \\
$h$ & $6 \cdot 10^{-4}$ & $\mathrm{~m}$ \\
$V_{f}$ & 1 & $\mathrm{~m} \mathrm{~s}^{-1}$ \\
$\rho$ & $10^{3}$ & $\mathrm{~kg} \mathrm{~m}^{-3}$ \\
$c_{p}$ & $10^{3}$ & $\mathrm{~J} \mathrm{~kg}^{-1} \mathrm{~K}^{-1}$ \\
$\lambda$ & $10^{-1}$ & $\mathrm{~W} \mathrm{~m}^{-1} \mathrm{~K}^{-1}$ \\
\hline
\end{tabular}

As a standard for explicit numerical calculations, we will use numerical values for $\lambda, V_{f}, \rho$, $c_{p}, R$ and $h$ as given in Table 1 . Based on these values, we find that $\lambda_{I} \approx 2.2 \cdot 10^{-2}$ and $\lambda_{I I} \approx 1.6 \cdot 10^{-4}$. Since $\delta=h / R$, we write $\lambda_{I}$ and $\lambda_{I I}$ as

$$
\lambda_{I}=\delta \hat{\lambda}, \quad \lambda_{I I}=\delta^{2} \hat{\lambda}, \quad \text { with } \quad \hat{\lambda}=\frac{\lambda R^{2}}{\rho c_{p} h^{3} V_{f}}
$$

where $\hat{\lambda}=5 / 3$. As $\delta=10^{-2} \ll 1$, we say that $\hat{\lambda}=O(1), \lambda_{I}=O(\delta)$, and $\lambda_{I I}=O\left(\delta^{2}\right)$, all with respect to the small parameter $\delta$. This means that both $\lambda_{I}$ and $\lambda_{I I}$ are small parameters and that we may use asymptotic techniques to find solutions for the temperatures $\hat{T}_{I}$ and $\hat{T}_{I I}$. In the zeroth-order approximation, we omit the terms containing $\lambda_{I}$ and $\lambda_{I I}$ in (13) and (16), respectively. This essentially amounts to neglecting the diffusive term with respect to the convective term. By doing this, we find the following differential equations for the zeroth-order approximations $\hat{T}_{I}^{0}$ and $\hat{T}_{I I}^{0}$ of $\hat{T}_{I}$ and $\hat{T}_{I I}$, respectively,

$$
\begin{aligned}
& \left(1-\hat{Y}^{2}\right) \frac{\partial \hat{T}_{I}^{0}}{\partial \hat{X}}=0 \\
& u \frac{\partial \hat{T}_{I I}^{0}}{\partial x}+v \frac{\partial \hat{T}_{I I}^{0}}{\partial y}=0 .
\end{aligned}
$$

These equations express that both $\hat{T}_{I}^{0}$ and $\hat{T}_{I I}^{0}$ are constant along the streamlines. Since $\hat{T}=1$ at the inlet of the mould and since all streamlines cross the inlet, this implies that 
$\hat{T}_{I}^{0}=\hat{T}_{I I}^{0}=1$ everywhere in the mould (then, of course, also $\hat{T}_{I I I}^{0}=1$ ). This solution does satisfy the boundary conditions at the inlet of the mould, and those at the flow front, but it does not satisfy the boundary conditions at the walls of the mould, where $\hat{T}_{I}=\hat{T}_{I I}=0$. Therefore, we expect that there are boundary layers near the walls where diffusion cannot be neglected with respect to convection. In the next three sections we shall investigate these boundary layers in the regions $G_{I}, G_{I I}$ and $G_{I I I}$, respectively. The fundamental small parameter, responsible for these boundary layers, is everywhere $\delta$. However, the size of the boundary layers is proportional to $\delta^{n}$, where $n$ will have different values in the three distinct regions.

\section{Boundary layer in $G_{I}$}

In this section, we consider the boundary layers near the walls $\hat{Y}= \pm 1$ within $G_{I}$. The fundamental small parameter, responsible for these boundary layers, is here $\lambda_{I}=O(\delta)$. It will turn out that here the size of the boundary layer is proportional to $\delta^{1 / 3}$.

\subsection{First-order solution}

Since the problem is symmetric with respect to $\hat{Y}$, we only have to consider the boundary layer near $\hat{Y}=1$. We introduce a new coordinate $\zeta$ defined by

$$
\hat{Y}=1-\epsilon \zeta \text {. }
$$

Here, $\epsilon$ is the, still unknown, thickness of the boundary layer, and $\zeta=O(1)$ in the boundary layer. Replacement of $\hat{Y}$ by $\zeta$ in (15) yields (omitting the hat in $\hat{X}$ )

$$
\epsilon \zeta(2-\epsilon \zeta) \frac{\partial T_{I}}{\partial X}=\frac{\lambda_{I}}{\epsilon^{2}} \frac{\partial^{2} T_{I}}{\partial \zeta^{2}} .
$$

Since we want the left-hand side of (24) to be of the same order as the right-hand side, $\epsilon$ must satisfy

$$
\epsilon^{3}=\lambda_{I}=O(\delta), \quad \Longrightarrow \quad \epsilon=\sqrt[3]{\lambda_{I}}=O\left(\delta^{1 / 3}\right) .
$$

With this choice for $\epsilon,(24)$ can be rewritten as

$$
2 \zeta \frac{\partial T_{I}}{\partial X}-\frac{\partial^{2} T_{I}}{\partial \zeta^{2}}=\epsilon \zeta^{2} \frac{\partial T_{I}}{\partial X}
$$

After neglecting terms of order $\epsilon$ in (26), we arrive at the following differential equation for the first-order asymptotic boundary layer solution in $G_{I}$, denoted by $T_{I}^{1}=T_{I}^{1}(X, \zeta)$,

$$
2 \zeta \frac{\partial T_{I}^{1}}{\partial X}-\frac{\partial^{2} T_{I}^{1}}{\partial \zeta^{2}}=0, \quad \text { for } \quad X>0, \zeta>0
$$

The boundary conditions for $T_{I}^{1}$ are given by

$$
T_{I}^{1}(0, \zeta)=1, \quad T_{I}^{1}(X, 0)=0 .
$$


In order that this boundary layer solution (or inner solution) matches with the zeroth-order solution (outer solution), we require that

$$
\text { for } \zeta \rightarrow \epsilon^{-1} \approx \infty: \quad T_{I}^{1} \rightarrow T_{I}^{0}=1 .
$$

To find the solution $T_{1}(X, \zeta)$ of (27) satisfying the conditions (28)-(29), we assume that there exists a similarity solution, which means that we assume that $T_{I}^{1}(X, \zeta)$ can be written as

$$
T_{I}^{1}(X, \zeta)=T_{I}^{1}(\sigma)
$$

with $\sigma=\zeta X^{\alpha}$, for a certain value for $\alpha$. Written in terms of $\sigma$, the partial differential equation (27) transforms into the ordinary differential equation for $T_{I}^{1}(\sigma)$ (after multiplication by $\left.X^{-2 \alpha}\right)$

$$
2 \alpha \zeta^{2} X^{-\alpha-1} \frac{d T_{I}^{1}}{d \sigma}-\frac{d^{2} T_{I}^{1}}{d \sigma^{2}}=0 .
$$

To be able to eliminate both $\zeta$ and $X$ from (31), $\zeta^{2} X^{-\alpha-1}$ must be a multiple of $\sigma$, implying that there must exist a $\beta \in \mathbb{R}$, such that

$$
\zeta^{2} X^{-\alpha-1}=\sigma^{\beta}=\zeta^{\beta} X^{\alpha \beta} .
$$

Equation (32) has exactly one solution for $\alpha$ and $\beta$, namely $\beta=2$ and $\alpha=-1 / 3$. Substitution of these values for $\alpha$ and $\beta$ into (31) yields

$$
\frac{2}{3} \sigma^{2} \frac{d T_{I}^{1}}{d \sigma}+\frac{d^{2} T_{I}^{1}}{d \sigma^{2}}=0 .
$$

Since $\alpha<0$, the boundary condition at $X=0$ and the matching condition for $\zeta \rightarrow \infty$ both imply a boundary condition for $T_{I}^{1}$ for $\sigma \rightarrow \infty$, which might lead to a contradiction. However, since $T_{I}^{1} \rightarrow 1$ for both $X=0$ and $\zeta \rightarrow \infty$, this is not the case here, and the conditions (28) and (29) transform into the boundary conditions for $T_{I}^{1}(\sigma)$ :

$$
T_{I}^{1}(0)=0, \quad \text { and } \quad T_{I}^{1}(\infty)=1 .
$$

Equation (33) can be integrated twice to yield the solution

$$
T_{I}^{1}(\sigma)=C \int_{0}^{\sigma} e^{-\frac{2}{9} s^{3}} d s+D
$$

where $C$ and $D$ are integration constants. From (34), it follows that $C$ and $D$ are equal to

$$
C=\left(\int_{0}^{\infty} e^{-\frac{2}{9} s^{3}} d s\right)^{-1}, \quad D=0,
$$

yielding the final solution for $T_{I}^{1}$, written in terms of $X$ and $\zeta$, in the form

$$
T_{I}^{1}(X, \zeta)=C \int_{0}^{\frac{\zeta}{\sqrt[3]{X}}} e^{-\frac{2}{9} s^{3}} d s .
$$

This result represents the first-order term (i.e. for $\epsilon \rightarrow 0$ ) of the inner solution in the boundary layer at $Y=1$. With this result, we can derive an expression for the thickness of the boundary 
layer in $G_{I}$. We define this thickness $h_{b l}=h_{b l}(X)$ as the value of $(h-Y)$ at which the scaled temperature $T_{I}$ differs $O(\epsilon)$ from the scaled inlet temperature, that is

$$
T_{I}\left(X, \zeta_{b l}\right)=1-\epsilon
$$

where $\zeta_{b l}=h_{b l} /(\epsilon h)$. For the determination (roughly in first order) of $\zeta_{b l}$, we use the firstorder solution (37), and we make use of the asymptotic expansion of the right-hand side of (37) for large values of $\zeta / \sqrt[3]{X}$, reading

$$
T_{I}\left(X, \zeta_{b l}\right)=1-e^{-\frac{2 \zeta^{3}}{9 X}},
$$

Equating (38) and (39), and eliminating $\zeta_{b l}$ in favour of $h_{b l}$, we obtain

$$
\frac{h_{b l}}{h} \approx \epsilon \sqrt[3]{\frac{9}{2} \hat{X} \log \left(\frac{1}{\epsilon}\right)} \approx \sqrt[3]{-2 \hat{\lambda} \delta \hat{X} \log \delta},
$$

where we have used (25) for $\epsilon$.

The second-order solution for $T_{I}$ (of $O(\epsilon)$ ) will be calculated in the next subsection. A comparison with numerical results will be presented in Section 3.3.

\subsection{Second-order solution}

The solution (37) is a first-order asymptotic boundary layer solution of (15). We can find a second-order solution, by assuming that the solution of (15) can be written as

$$
T_{I}(X, Y)=T_{I}(X, 1-\epsilon \zeta)=T_{I}^{1}(X, \zeta)+\epsilon T_{I}^{2}(X, \zeta)+O\left(\epsilon^{2}\right)
$$

where $T_{I}^{2}(X, \zeta)=O(1)$ for $\zeta=O(1)$. Substituting (41) into (17), using (37) and neglecting terms of order $\epsilon^{2}$, we obtain

$$
2 \zeta \frac{\partial T_{I}^{2}}{\partial X}-\frac{\partial^{2} T_{I}^{2}}{\partial \zeta^{2}}=-C \frac{\zeta^{3}}{3 X^{4 / 3}} e^{-\frac{2 \zeta^{3}}{9 X}}
$$

The boundary conditions for $T_{I}^{2}$ are

$$
T_{I}^{2}(X, 0)=0, \quad T_{I}^{2}(0, \zeta)=0,
$$

and

$$
T_{I}^{2}(0, \zeta) \rightarrow 0, \quad \text { for } \quad \zeta \rightarrow \infty
$$

Analogous to the first-order solution, we will try to find a similarity solution of (42). We assume that $T_{I}^{2}$ can be written as $T_{I}^{2}(X, \zeta)=\zeta F(\sigma)$, where $\sigma=\zeta / \sqrt[3]{X}$. With this assumption, the partial differential equation (42) turns into an ordinary differential equation for $F(\sigma)$ (after multiplication by $-\sqrt[3]{X})$

$$
\left(\frac{2}{3} \sigma^{3}+2\right) F^{\prime}(\sigma)+\sigma F^{\prime \prime}(\sigma)=\frac{C}{3} \sigma^{3} e^{-\frac{2}{9} \sigma^{3}} .
$$

The differential equation (45) is a quasi-linear ordinary differential equation. Therefore, the general solution of (45) can be composed of the solution of the homogeneous system plus a 
particular solution, where the latter can be found from the first by the method of variation of constants. This leads to

$$
F(\sigma)=C_{1} \int_{\sigma}^{\infty} \frac{1}{s^{2}} e^{-\frac{2}{9} s^{3}} d s+C_{2}+\frac{C}{15} \int_{0}^{\sigma} s^{3} e^{-\frac{2}{9} s^{3}} d s=-\frac{C}{15} \int_{\sigma}^{\infty} s^{3} e^{-\frac{2}{9} s^{3}} d s,
$$

because $F(0)$ must be finite $\left(\Rightarrow C_{1}=0\right)$ and $F(\sigma)$ should go to zero at infinity (this determines $\left.C_{2}\right)$.

Thus, the second-order asymptotic boundary layer solution $T_{I}^{2}$ is given by

$$
T_{I}^{2}(X, \zeta)=-\frac{C \zeta}{15} \int_{\frac{\zeta}{\sqrt[3]{X}}}^{\infty} s^{3} e^{-\frac{2}{9} s^{3}} d s
$$

\subsection{Numerical results and comparison of first-order and second-order so- lution}

We compare the asymptotic solutions $T_{I}^{1}(X, \zeta)$ and $T_{I}^{1}(X, \zeta)+\epsilon T_{I}^{2}(X, \zeta)$, where $T_{I}^{1}$ and $T_{I}^{2}$ are given by given by (37) and (47), respectively, with a numerical solution of (15) calculated by a finite-difference method. In Table 2, the numerical results are presented for a scheme of 10 grid points in both $X$ - and $Y$-direction. In this table, $T_{1}, T_{2}, \Delta T_{1}$ and $\Delta T_{2}$ correspond to

$$
\begin{array}{ll}
T_{1}(X, Y)=T_{I}^{1}\left(X, \frac{1-Y}{\epsilon}\right), & T_{2}(X, Y)=\left(T_{I}^{1}+\epsilon T_{I}^{2}\right)\left(X, \frac{1-Y}{\epsilon}\right), \\
\Delta T_{1}=\frac{T_{1}-T_{\text {num }}}{T_{\text {num }}}, & \Delta T_{2}=\frac{T_{2}-T_{\text {num }}}{T_{\text {num }}},
\end{array}
$$

where we have chosen for $X$ the value $X=1$. In dimensional variables, this corresponds with $X=6 \cdot 10^{-2} \mathrm{~m}$. Moreover, we have taken $\lambda_{I}=0.01$, corresponding to a value of $\epsilon=\sqrt[3]{\lambda_{I}}=0.215$.

Table 2: Comparison between asymptotic and numerical results at $X=1$

\begin{tabular}{|l|l|l|l|l|l|}
\hline$Y$ value & \multicolumn{2}{|l|}{ Asymptotic solution } & Num. sol. & \multicolumn{2}{c|}{ Relative difference } \\
& $T_{1}$ & $T_{2}$ & $T_{\text {num }}$ & $\Delta T_{1}$ & $\Delta T_{2}$ \\
\hline 0.2 & 1. & 1. & 1. & 0 & 0 \\
0.4 & 0.9990 & 0.9980 & 0.9974 & 0.0016 & 0.0006 \\
0.6 & 0.9465 & 0.9322 & 0.9303 & 0.0174 & 0.0020 \\
0.7 & 0.8241 & 0.8033 & 0.7989 & 0.0315 & 0.0054 \\
0.8 & 0.6031 & 0.5846 & 0.5775 & 0.0442 & 0.0122 \\
0.9 & 0.3131 & 0.3032 & 0.2974 & 0.0528 & 0.0193 \\
\hline
\end{tabular}

From Table 2, it follows that the asymptotic solutions and the numerical solution are in good agreement with each other. This despite the fact that the parameter $\epsilon=\sqrt[3]{\lambda}=0.215$ is not really very small. The relative differences between the numerical solution and the first-order and second-order asymptotic solutions are of the same order, and are in fact only of some 
relevance very near to the walls at $Y= \pm 1$. We conclude that for practical purposes the first-order asymptotic solution suffices. This is confirmed by the graphs for $T_{I}^{1}$ and $T_{I}^{2}$ shown in Figure 2 a. and b., respectively, where for $X$ the value $X=0.5$ is chosen. From Figure 2 a. we see that the boundary layer here reaches to $\zeta \approx 2$; for $\zeta>2$ the temperature is equal to the inlet temperature, $T_{I}=1$. Moreover, Figure $2 \mathrm{~b}$. reveals that the second-order correction $T_{I}^{2}=\left(T_{1}-T_{2}\right) / \epsilon$ is maximal somewhere in the middle of the boundary layer, but even there the total correction remains below $1 \%$ of the inlet temperature. Hence, for practical purposes the first-order approximation suffices.

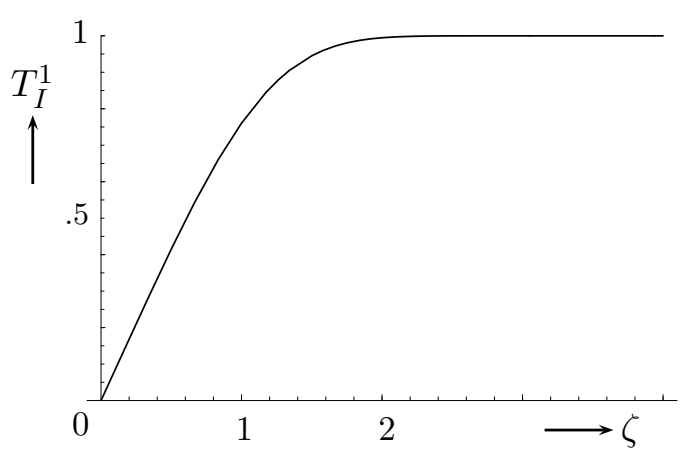

a.

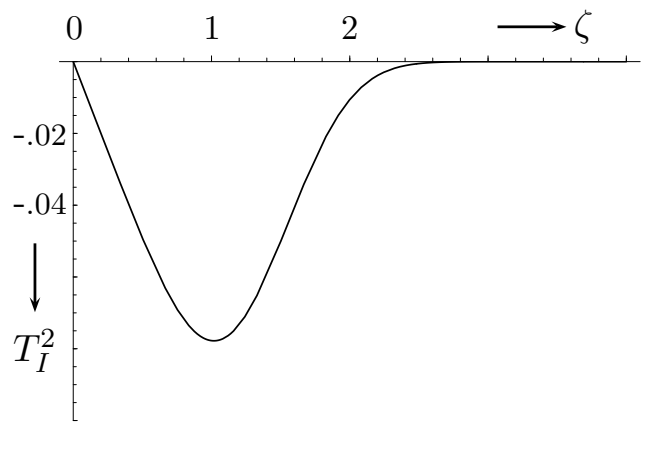

b.

Figure 2: a. The first-order solution $T_{I}^{1}$ as a function of $\zeta$ for $\hat{X}=0.5$; b. the second-order solution $T_{I}^{2}$ as a function of $\zeta$ for $\hat{X}=0.5$.

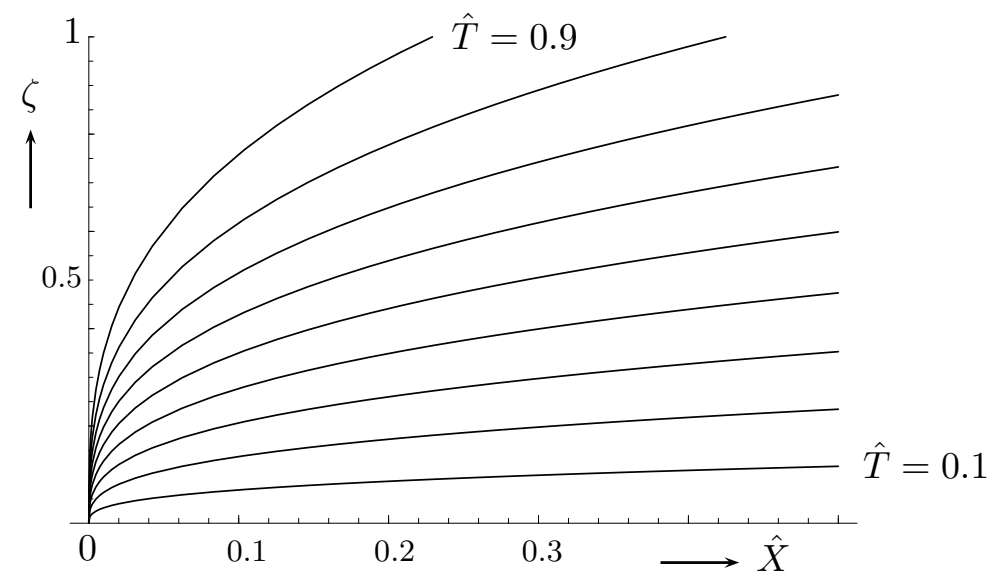

Figure 3: A set of isothermals for $T_{I}^{1}$ in the $(\hat{X}, \zeta)$-plane in the region $G_{I}$ far behind the flow front region.

In Figure 3, we have depicted a set of isothermals, ranging from $\hat{T}=0.9$ to $\hat{T}=0.1$, in the $\hat{X}-\zeta$-plane in the region $G_{I}$, so near the upper wall (where $\hat{T}=0$ ). We observe that the isothermals initially, that is for small $\hat{X}$-values, behave rather steeply; this is due to the large difference between the inlet temperature and the wall temperature. However, they strongly flatten for increasing $\hat{X}$. The major changes in $\hat{T}$ in the vertical or $Y$-direction take place within the $O(\epsilon)$-range $0<\zeta<1$ (the boundary layer) near the wall. 


\section{Boundary layer in $G_{I I}$}

In this section, we assume boundary layers in the upper and lower parts of the flow front region $G_{I I}$ near the points $x=0, y= \pm 1$. The fundamental small parameter, responsible for these boundary layers, is here $\lambda_{I I}=\delta^{2} \hat{\lambda}=O\left(\delta^{2}\right)$.

\subsection{Temperature problem in the boundary layer in $G_{I I}$}

Since the problem is symmetric with respect to $y$, we only have to consider the boundary layer near $y=1$. We introduce a new coordinate system $(\sigma, \tau)$, where $\sigma$ and $\tau$ are defined by

$$
x=-\epsilon_{1} \sigma, \quad y=1-\epsilon_{2} \tau .
$$

Here, $\epsilon_{1}$ and $\epsilon_{2}$ are, still unknown, characteristic measures for the thicknesses of the boundary layer in the $x$ - and $y$-directions, respectively (see Figure 4 ), where $0<\epsilon_{1}, \epsilon_{2} \ll 1$. To determine $\epsilon_{1}$ and $\epsilon_{2}$, we substitute (49) into (16), and choose $\epsilon_{1}$ and $\epsilon_{2}$ such that the leading order terms in the resulting differential equation include both diffusive and convective terms.

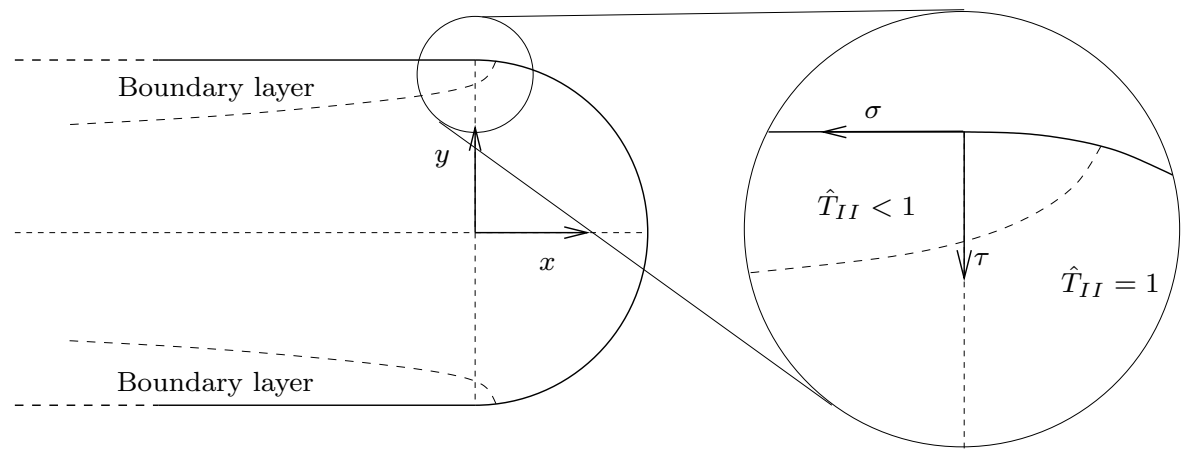

Figure 4: Global and detailed view of boundary layer near the point $(x, y)=(0,1)$.

Substitution of (49) into (16) yields the following differential equation for the temperature in the boundary layer:

$$
\frac{\bar{u}}{\epsilon_{1}} \frac{\partial \bar{T}}{\partial \sigma}+\frac{\bar{v}}{\epsilon_{2}} \frac{\partial \bar{T}}{\partial \tau}=\frac{\lambda_{I I}}{\epsilon_{1}^{2}} \frac{\partial^{2} \bar{T}}{\partial \sigma^{2}}+\frac{\lambda_{I I}}{\epsilon_{2}^{2}} \frac{\partial^{2} \bar{T}}{\partial \tau^{2}},
$$

where

$$
\begin{aligned}
& \bar{T}(\sigma, \tau)=\hat{T}_{I I}\left(-\epsilon_{1} \sigma, 1-\epsilon_{2} \tau\right), \\
& \bar{u}(\sigma, \tau)=-u\left(-\epsilon_{1} \sigma, 1-\epsilon_{2} \tau\right), \\
& \bar{v}(\sigma, \tau)=-v\left(-\epsilon_{1} \sigma, 1-\epsilon_{2} \tau\right) .
\end{aligned}
$$

For $\sigma=O(1)$ and $\tau=O(1), \bar{u}$ may be approximated using a Taylor series expansion around $(\sigma, \tau)=(0,0)$ according to (here, h.o.t. stands for higher-order terms)

$$
\begin{aligned}
\bar{u}(\sigma, \tau) & =\bar{u}(0,0)+\sigma \frac{\partial \bar{u}}{\partial \sigma}(0,0)+\tau \frac{\partial \bar{u}}{\partial \tau}(0,0)+\text { h.o.t. } \\
& =1+\epsilon_{1} \sigma u_{, x}(0,1)+\epsilon_{2} \tau v_{, y}(0,1)+\text { h.o.t. }
\end{aligned}
$$


while, analogously, $\bar{v}$ can be approximated as

$$
\bar{v}(\sigma, \tau)=\epsilon_{1} \sigma v_{, x}(0,1)+\epsilon_{2} \tau v_{, y}(0,1)+\text { h.o.t. }
$$

where we used the fact that $\mathbf{u}(0,1)=(-1,0)$. Assuming that $|\nabla \mathbf{u}(0,1)|=O(1)$, we obtain the following approximation for $\overline{\mathbf{u}}$

$$
\bar{u}(\sigma, \tau)=1+O\left(\epsilon_{1}, \epsilon_{2}\right), \quad \bar{v}(\sigma, \tau)=O\left(\epsilon_{1}, \epsilon_{2}\right) .
$$

After substitution of (54) into (50), we find the differential equation for the temperature in the boundary layer:

$$
\underbrace{\frac{\partial \bar{T}}{\partial \sigma}}_{I}=\underbrace{\frac{\lambda_{I I}}{\epsilon_{1}} \frac{\partial^{2} \bar{T}}{\partial \sigma^{2}}}_{I I}+\underbrace{\frac{\lambda_{I I} \epsilon_{1}}{\epsilon_{2}^{2}} \frac{\partial^{2} \bar{T}}{\partial \tau^{2}}}_{I I I}+\frac{\epsilon_{1}}{\epsilon_{2}} O\left(\epsilon_{1}, \epsilon_{2}\right),
$$

In this differential equation, term $I$ is of $O(1)$. Therefore, we get the 'richest' differential equation, if both terms $I I$ and $I I I$ are of $O(1)$ too, resulting in the following choice for $\epsilon_{1}$ and $\epsilon_{2}$ :

$$
\epsilon_{1}=\epsilon_{2}=2 \lambda_{I I}=2 \delta^{2} \hat{\lambda}=: \epsilon \ll 1,
$$

so $\epsilon$ is proportional to $\delta^{2}$ here.

This renders the following differential equation for $\bar{T}$, which is valid in the boundary layer at the upper part of the flow front

$$
\frac{\partial^{2} \bar{T}}{\partial \sigma^{2}}+\frac{\partial^{2} \bar{T}}{\partial \tau^{2}}-2 \frac{\partial \bar{T}}{\partial \sigma}=0, \quad \text { for } \quad \tau>0
$$

For a complete formulation of the temperature problem for $\bar{T}$, we still need the boundary conditions for (57), which follow from (18)-(19). Moreover, we will need matching conditions to match the boundary layer solutions in $G_{I I}$ to the zeroth-order solution in $G_{I I}$ (i.e. $\bar{T}=1$ ). We start with the boundary conditions.

- The boundary $y=1, x<0$ corresponds to $\tau=0, \sigma>0$. Hence, $\bar{T}(\sigma, 0)=0$, for all $\sigma>0$.

- The boundary $x=x_{f}(y)$, for $0<y<1, x>0$, i.e. the flow front, corresponds to

$$
-\epsilon \sigma=x_{f}(1-\epsilon \tau) \quad \text { and } \quad 0 \leq \tau \leq \frac{1}{\epsilon} .
$$

Since $x_{f}(\zeta)$ is a monotone decreasing function in $\zeta$ for $0<\zeta<1$, the equation for the upper half of the flow front may be rewritten as

$$
\epsilon \tau=1-x_{f}^{-1}(-\epsilon \sigma), \quad \text { for } \quad \epsilon \sigma<0,
$$

where $x_{f}^{-1}$ is the inverse function of $x_{f}$. Using a Taylor series expansion for $x_{f}^{-1}(x)$ in the neighbourhood of $x=0$, we derive the following implicit equation for the flow front within the boundary layer in terms of $\sigma$ and $\tau$

$$
\begin{aligned}
\tau & =\frac{1}{\epsilon}\left(1-x_{f}^{-1}(0)+\left(x_{f}^{-1}\right)^{\prime}(0) \epsilon \sigma-\frac{1}{2}\left(x_{f}^{-1}\right)^{\prime \prime}(0) \epsilon^{2} \sigma^{2}+O\left(\epsilon^{3}\right)\right) \\
& =\left(x_{f}^{-1}\right)^{\prime}(0) \sigma-\frac{1}{2}\left(x_{f}^{-1}\right)^{\prime \prime}(0) \epsilon \sigma^{2}+O\left(\epsilon^{2}\right),
\end{aligned}
$$


holding for all $\sigma<0$, as long as $\sigma=O(1)$ for $\epsilon \rightarrow 0$. Here, we used the fact that $x_{f}^{-1}(0)=x_{f}^{-1}\left(x_{f}(1)\right)=1$.

Since the flow front is tangent to the wall in the point $(\sigma, \tau)=(0,0),\left(x_{f}^{-1}\right)^{\prime}(0)=0$. Using this, (4.1) indicates that in the boundary layer, $\tau=O(\epsilon)$, for $\sigma<0$.

The boundary condition at the flow front following from (18) can be written as

$$
\frac{\partial \bar{T}}{\partial \tau}=\frac{1}{x_{f}^{\prime}(1-\epsilon \tau)} \frac{\partial \bar{T}}{\partial \sigma}
$$

Since

$$
\frac{1}{x_{f}^{\prime}(1-\epsilon \tau)}=\left(x_{f}^{-1}\right)^{\prime}(-\epsilon \sigma)=O(\epsilon)
$$

and since $\epsilon$ is chosen such that $\partial \bar{T} / \partial \sigma$ is finite, the boundary condition at the flow front reduces to

$$
\frac{\partial \bar{T}}{\partial \tau}=O(\epsilon)
$$

- The solution in the boundary layer must match with the inner solution $(T \approx 1)$. Therefore, we require that for all $\sigma \in \mathbb{R}, \bar{T}(\sigma, \tau) \rightarrow 1$ for $\tau \rightarrow 1 / \epsilon$. Moreover, we assume that $\bar{T}(\sigma, \tau)=O(1)$ for all $\sigma$ and for all $\tau \geq 0$.

\subsection{First-order solution}

In the previous sections, we derived the differential equation for the temperature in the boundary layer in $G_{I I}$, together with the pertinent boundary conditions, for small $\epsilon$. For the first-order solution, we let $\epsilon \rightarrow 0$, which leads us to the following problem for $\bar{T}(\sigma, \tau)$ in the half plane $\tau>0$ :

$$
\begin{aligned}
& \frac{\partial^{2} \bar{T}}{\partial \sigma^{2}}+\frac{\partial^{2} \bar{T}}{\partial \tau^{2}}-2 \frac{\partial \bar{T}}{\partial \sigma}=0, \tau>0, \quad \sigma \in \mathbb{R} ; \\
& \bar{T}(\sigma, 0)=0, \quad \text { for } \quad \sigma>0, \text { for } \quad \sigma<0, \\
& \frac{\partial \bar{T}}{\partial \tau}(\sigma, 0)=0, \quad \text { for } \quad \tau \rightarrow \infty, \quad \sigma \in \mathbb{R},
\end{aligned}
$$

We replace $\bar{T}$ by a new function $\phi=\phi(\sigma, \tau)$, defined according to

$$
\phi(\sigma, \tau)=e^{-\sigma}(1-\bar{T}(\sigma, \tau)),
$$

which goes sufficiently fast to zero for $\zeta \rightarrow \pm \infty$, to assure that its Fourier transform

$$
\Phi(w, \tau)=\mathfrak{F}(\phi(\sigma, \tau) ; \sigma \rightarrow w)=\int_{-\infty}^{\infty} \phi(\sigma, \tau) e^{i w \sigma} d \sigma
$$

for $w \in \mathbb{C}$ exists.

From this definition, it follows that $\phi(\sigma, \tau)$ satisfies the following differential equation, valid on the half-plane $\tau \geq 0, \sigma \in \mathbb{R}$

$$
\frac{\partial^{2} \phi}{\partial \sigma^{2}}+\frac{\partial^{2} \phi}{\partial \tau^{2}}=\phi
$$


together with the boundary conditions

$$
\begin{aligned}
& \phi(\sigma, 0)=e^{-\sigma}, \quad \text { for } \quad \sigma>0 \\
& \frac{\partial \phi}{\partial \tau}(\sigma, 0)=0, \quad \text { for } \quad \sigma<0
\end{aligned}
$$

and

$$
\phi(\sigma, \tau) \rightarrow 0, \quad \text { for } \quad \tau \rightarrow \infty, \quad \sigma \in \mathbb{R} .
$$

The system (69)-(72) can be solved by using a Wiener-Hopf technique along the same lines as presented by Carrier et al. [14, Section 8-1]. This results in the following expression for $\Phi(w, \tau)$ (for the details of the derivation, we refer to [11])

$$
\Phi(w, \tau)=\frac{1+i}{(w+i) \sqrt{w-i}} e^{-\tau \sqrt{w^{2}+1}}
$$

which is valid for $\tau>0$ and $-1<\operatorname{Im}(w)<1$.

This result should be compared to the second equation of (8-12) in [14]; in fact, it can be obtained from the latter equation by letting $a$ go to zero, and replacing $y$ by $2 \sigma$ and $\lambda$ by $(\lambda+i) / 2$ (the reason for the latter is that we have to replace our function $\phi$ by $e^{\sigma} \phi$ in order to get the same problem as in [14], and this leads to a shift in $\lambda$ in the Fourier transform).

To find $\phi(\sigma, \tau)$, we apply an inverse Fourier transformation on $\Phi(w, \tau)$ with respect to $w$, i.e.

$$
\phi(\sigma, \tau)=\frac{1}{2 \pi} \int_{-\infty+i \delta}^{\infty+i \delta} \Phi(w, \tau) e^{-i \sigma w} d w
$$

with $-1<\delta<1$. From (73) and the definitions of $\sqrt{w^{2}+1}$ and $\sqrt{w-i}$, it follows that for all $\tau \geq 0, \Phi(w, \tau)$ is analytic in $\mathbb{C}$ excluding cuts from $i$ to $i \infty$ and from $-i$ to $-i \infty$ along the imaginary axis. Therefore, in order to find a closed form for $\phi(\sigma, \tau)$, we may use contour integration. Since $\operatorname{Re}(-i w)>0$ for $\operatorname{Im}(w)>0$, and vice versa, we choose a closed contour $C$ in the upper half of the $w$-plane to calculate $\phi(\sigma, \tau)$ for $\sigma<0$, and a closed contour $D$ in the lower half of the $w$-plane to calculate $\phi(\sigma, \tau)$ for $\sigma>0$, excluding the cuts from $\pm i$ to $\pm i \infty$ along the imaginary axis. More specific, we choose $C$ and $D$ to be the boundaries of $G^{+}$and $G^{-}$, respectively, where $G^{ \pm}$are given by

$$
\begin{aligned}
& G^{+}:=\left\{z \in \mathbb{C}\left|\operatorname{Im}(z)>\delta_{1} \wedge\right| z|<R \wedge| z-i \mid>\epsilon \wedge z \notin I^{+}\right\}, \quad C=\partial G^{+}, \\
& G^{-}:=\left\{z \in \mathbb{C}\left|\operatorname{Im}(z)<-\delta_{2} \wedge\right| z|<R \wedge| z+i \mid>\epsilon \wedge z \notin I^{-}\right\}, \quad D=\partial G^{-},
\end{aligned}
$$

with

$$
\begin{gathered}
I^{+}:=\{z \in \mathbb{C} \mid \operatorname{Im}(z) \geq 1 \wedge \operatorname{Re}(z)=0\} \\
I^{-}:=\{z \in \mathbb{C} \mid \operatorname{Im}(z) \leq-1 \wedge \operatorname{Re}(z)=0\}
\end{gathered}
$$

In (75) and (76), $\delta_{1}$ and $\delta_{2}$ are arbitrary constants between 0 and 1 , while $R$ and $\epsilon$ are positive constants such that $R \gg 1$ and $1-\epsilon>\left(\delta_{1}, \delta_{2}\right)$. By taking limits for $R \rightarrow \infty$ and $\epsilon \rightarrow 0$, we will derive a closed-form expression for $\phi(\sigma, \tau)$. To calculate this expression, we split $C$ and $D$ into six disjoint parts:

$$
C=C_{1} \cup C_{2} \cup C_{3} \cup C_{4} \cup C_{5} \cup C_{6}, \quad D=D_{1} \cup D_{2} \cup D_{3} \cup D_{4} \cup D_{5} \cup D_{6},
$$




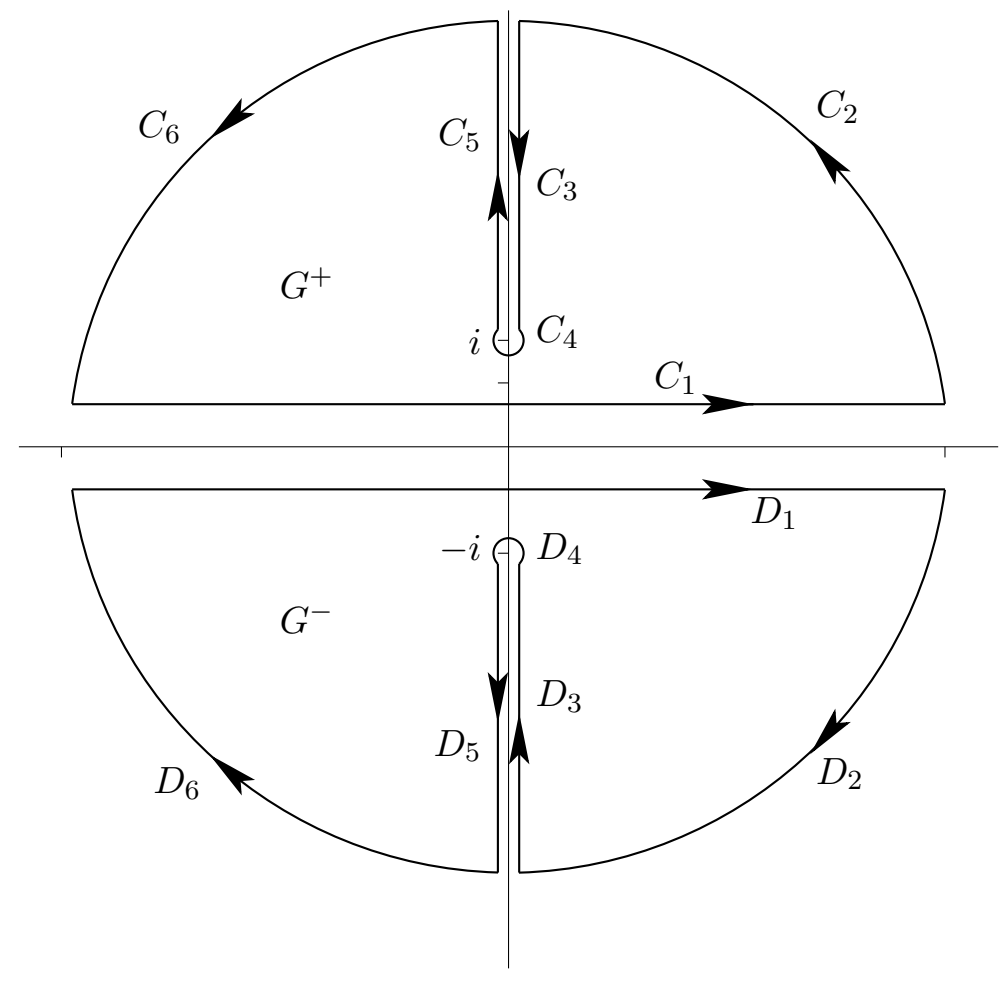

Figure 5: Contours $C$ and $D$ in $w$-plane.

as shown in Figure 5.

First, consider the case where $\sigma>0$. Then, $\delta \rightarrow-\delta_{2}$ in (74), and thus

$$
\phi(\sigma, \tau)=\frac{1}{2 \pi} \lim _{R \rightarrow \infty} \int_{D_{1}} \Phi(w, \tau) e^{-i \sigma w} d w .
$$

Since $\Phi(w, \tau)$ is analytic in $G^{-}$, the contour integral along $D$ must be zero, so

$$
\int_{D_{1}} \Phi(w, \tau) e^{-i \sigma w} d w=-\int_{D_{2} \cup D_{3} \ldots \cup D_{6}} \Phi(w, \tau) e^{-i \sigma w} d w .
$$

Since $\operatorname{Re}\left(\sqrt{w^{2}+1}\right) \geq 0$ for all $w$ for which it is defined, it follows from (73) that for all $\tau>0$, $\Phi(w, \tau)=O\left(R^{-3 / 2}\right)$ for $|w|=R \rightarrow \infty$. Therefore, from Jordan's lemma (see [14, p. 81-82]) it follows that

$$
\lim _{R \rightarrow \infty} \int_{D_{2} \cup D_{6}} \Phi(w, \tau) e^{-i \sigma w} d w=0 .
$$

In the half-plane $\operatorname{Im} w<0, \Phi(w, \tau)$ has one singular point, being a pole of order 1 in $z=-i$. Therefore,

$$
\lim _{\epsilon \rightarrow 0} \int_{D_{4}} \Phi(w, \tau) e^{-i \sigma w} d w=2 \pi i \operatorname{Res}_{w=-i} \Phi(w, \tau) e^{-i \sigma w}=-2 \pi e^{-\sigma} .
$$

Finally, we have to integrate $\Phi$ along $D_{3}$ and $D_{5}$, where $w=-i v \pm i \epsilon$, respectively, with $v>1$, and $0<\epsilon \ll 1$. Using that $\operatorname{Re}\left(\sqrt{w^{2}+1}\right) \geq 0$, for all $w$, we deduce that for $w \in D_{3} \cup D_{5}$,

$$
\sqrt{w^{2}+1}=\sqrt{(-i v \pm i \epsilon)^{2}+1} \approx \mp i \sqrt{v^{2}-1}\left(1 \mp \frac{v}{v^{2}-1} \epsilon\right)
$$


yielding, for $\epsilon \rightarrow 0$,

$$
\sqrt{w^{2}+1}=\mp i \sqrt{v^{2}-1}
$$

for $w \in D_{3}$ or $w \in D_{5}$, respectively. Analogously, we deduce

$$
\sqrt{w-i}=\frac{1-i}{\sqrt{2}} \sqrt{v+1}
$$

for $w \in D_{3} \cup D_{5}$.

From (85) and (86), and with $\Phi$ according to (73), it follows that

$$
\begin{aligned}
\int_{D_{3}} \Phi(w, \tau) e^{-i \sigma w} d w & =i \sqrt{2} \int_{R}^{1+\epsilon} \frac{e^{i \tau \sqrt{v^{2}-1}}}{(v-1) \sqrt{v+1}} e^{-\sigma v} d v= \\
& =-i \sqrt{2} \int_{\sqrt{2 \epsilon+\epsilon^{2}}}^{\infty} \frac{e^{-\sigma \sqrt{x^{2}+1}}}{\sqrt{x^{2}+1} \sqrt{\sqrt{x^{2}+1}-1}} e^{i \tau x} d x
\end{aligned}
$$

where we used the coordinate transformation $v=\sqrt{x^{2}+1}$, and where we let $R \rightarrow \infty$.

Similarly, the integral along $D_{5}$ is given by

$$
\int_{D_{5}} \Phi(w, \tau) e^{-i \sigma w} d w=i \sqrt{2} \int_{\sqrt{2 \epsilon+\epsilon^{2}}}^{\infty} \frac{e^{-\sigma \sqrt{x^{2}+1}}}{\sqrt{x^{2}+1} \sqrt{\sqrt{x^{2}+1}-1}} e^{-i \tau x} d x .
$$

Summing up the integrals in (87) and (88), and letting $\epsilon \rightarrow 0$, we find

$$
\int_{D_{3} \cup D_{5}} \Phi(w, \tau) e^{-i \sigma w} d w=2 \sqrt{2} \int_{0}^{\infty} \frac{e^{-\sigma \sqrt{x^{2}+1}}}{\sqrt{x^{2}+1} \sqrt{\sqrt{x^{2}+1}-1}} \sin (\tau x) d x .
$$

Note that although the integrals in (87) and (88) are divergent for $\epsilon \downarrow 0$, the integral in (89) does converge for $x \rightarrow 0$. Recollecting all the results derived above, we thus obtain for $\sigma>0$,

$$
\phi(\sigma, \tau)=e^{-\sigma}-\frac{\sqrt{2}}{\pi} \int_{0}^{\infty} \frac{e^{-\sigma \sqrt{x^{2}+1}}}{\sqrt{x^{2}+1} \sqrt{\sqrt{x^{2}+1}-1}} \sin (\tau x) d x .
$$

Next, we consider the case where $\sigma<0$. Analogous to the previous case, we can derive

$$
\phi(\sigma, \tau)=\frac{\sqrt{2}}{\pi} \int_{0}^{\infty} \frac{e^{\sigma \sqrt{x^{2}+1}}}{\sqrt{x^{2}+1} \sqrt{\sqrt{x^{2}+1}+1}} \cos (\tau x) d x
$$

for $\sigma<0$.

The integrals in (90) and (91) may be interpreted as Fourier transforms with respect to $x$. In [15] it can be found that $\phi(\sigma, \tau)$ for both $\sigma \geq 0$ and $\sigma<0$ is given by

$$
\phi(\sigma, \tau)=e^{-\sigma} \operatorname{Erfc}\left(\sqrt{\sqrt{\sigma^{2}+\tau^{2}}-\sigma}\right) .
$$

From (67), it then follows that $\bar{T}(\sigma, \tau)$ is equal to

$$
\bar{T}(\sigma, \tau)=1-e^{\sigma} \phi(\sigma, \tau)=\operatorname{Erf}\left(\sqrt{\sqrt{\sigma^{2}+\tau^{2}}-\sigma}\right) .
$$


Here, $\operatorname{Erf}(z)$ and $\operatorname{Erfc}(z)$ are the error function and the complementary error function, defined as

$$
\operatorname{Erf}(z)=\frac{2}{\sqrt{\pi}} \int_{0}^{z} e^{-s^{2}} d s, \quad \operatorname{Erfc}(z)=1-\operatorname{Erf}(z)
$$

respectively.

At this point, we have derived a solution for $\bar{T}(\sigma, \tau)$. It is easily checked that this solution indeed satisfies all the equations and conditions of system (63)-(66).

\section{Similarity Solution}

The specific form of the solution (93) suggests that, just as in (30), there exists a similarity solution in which $\bar{T}(\sigma, \tau)$ is only a function of $\rho=\rho(\sigma, \tau)=\sqrt{\sqrt{\sigma^{2}+\tau^{2}}-\sigma}$. To show that this is indeed true, we start from (63), which we first transform to cylindrical coordinates by taking

$$
\sigma=r \cos \varphi, \quad \tau=r \sin \varphi, \quad \varphi \in[0, \pi]
$$

yielding for $T=\tilde{T}(r, \varphi)$

$$
\frac{1}{r} \frac{\partial}{\partial r}\left(r \frac{\partial \tilde{T}}{\partial r}\right)+\frac{1}{r^{2}} \frac{\partial^{2} \tilde{T}}{\partial \varphi^{2}}-2 \cos \varphi \frac{\partial \tilde{T}}{\partial r}+\frac{2 \sin \varphi}{r} \frac{\partial \tilde{T}}{\partial \varphi}=0
$$

Expressing $\rho$ in terms of $r$ and $\varphi$, we obtain

$$
\rho=\tilde{\rho}(r, \varphi)=\sqrt{r-r \cos \varphi}=\sqrt{2} r^{1 / 2} \sin (\varphi / 2) .
$$

Assuming that $\tilde{T}(r, \varphi)=T(\rho)$, and using this in (96), we arrive after some elementary calculations at

$$
\frac{1}{2 r}\left[T^{\prime \prime}(\rho)+2 \rho T^{\prime}(\rho)\right]=0 .
$$

This ordinary differential equation has the general solution

$$
T(\rho)=c_{1} \int_{0}^{\rho} e^{-t^{2}} d t+c_{2} .
$$

The boundary conditions (64) and (65) can be written as

- for $\sigma \geq 0$,

$$
\bar{T}(\sigma, 0)=\tilde{T}(r, 0)=T(0)=c_{2}=0 ;
$$

- for $\sigma<0$,

$$
\frac{\partial \bar{T}}{\partial \tau}(\sigma, 0)=-\frac{1}{r} \frac{\partial \tilde{T}}{\partial \varphi}(r, \pi)=0, \quad \text { (identically) }
$$

- for $\sigma \in \mathbb{R}, \tau \rightarrow \infty$,

$$
\bar{T}(\sigma, \tau) \rightarrow \tilde{T}(r \rightarrow \infty, \varphi) \rightarrow T(\rho \rightarrow \infty) \rightarrow \frac{\sqrt{\pi}}{2} c_{1}=1 .
$$


Hence, $c_{1}=2 / \sqrt{\pi}$ and $c_{2}=0$, rendering our previous result (93).

\section{Thickness of boundary layer}

We can use the here obtained similarity solution for the determination of the thickness of the boundary layer in $G_{I I}$. Let $\rho_{b l}$ be the value of $\rho$ at the border of the boundary layer. Then, we find $\rho_{b l}$ from $\operatorname{Erfc}\left(\rho_{b l}\right)=\epsilon$, resulting in

$$
\rho_{b l}=\sqrt{\log (1 / \epsilon)}(1+o(1)) \approx \sqrt{-2 \log \delta} .
$$

Once $\rho_{b l}$ is known, we find the dimensionless thickness $\tau_{b l}$ of the boundary layer from $\sqrt{\sigma^{2}+\tau_{b l}^{2}}-\sigma=\rho_{b l}^{2}$, yielding with $h_{b l}=2 h \hat{\lambda} \delta^{2} \tau_{b l}$

$$
\frac{h_{b l}}{h}=2 \hat{\lambda} \delta^{2} \rho_{b l}^{2} \sqrt{1+\frac{2 \sigma}{\rho_{b l}^{2}}} \approx-4 \hat{\lambda} \delta^{2} \log \delta \sqrt{1-\frac{\sigma}{\log \delta}} .
$$

From this, we see that $h_{b l} \downarrow 0$ for $\sigma \rightarrow-\rho_{b l}^{2} / 2 \approx \log \delta$. This $\sigma$-value corresponds to $x=$ $-2 \hat{\lambda} \delta^{2} \log \delta$, and indicates the right end point of the boundary layer in $G_{I I}$.

Moreover, if $\sigma \gg \rho_{b l}^{2} \sim \log (1 / \delta)$ then

$$
\frac{h_{b l}}{h} \sim 2 \hat{\lambda} \delta^{2} \rho_{b l} \sqrt{2 \sigma} \approx 4 \hat{\lambda} \delta^{2} \sqrt{-\sigma \log \delta}=2 \sqrt{2 \hat{\lambda}} \delta \sqrt{\frac{V_{f} t-X}{h}} \sqrt{-\log \delta},
$$

where the latter step follows from (49), (56) and (4).

\subsection{Numerical results}

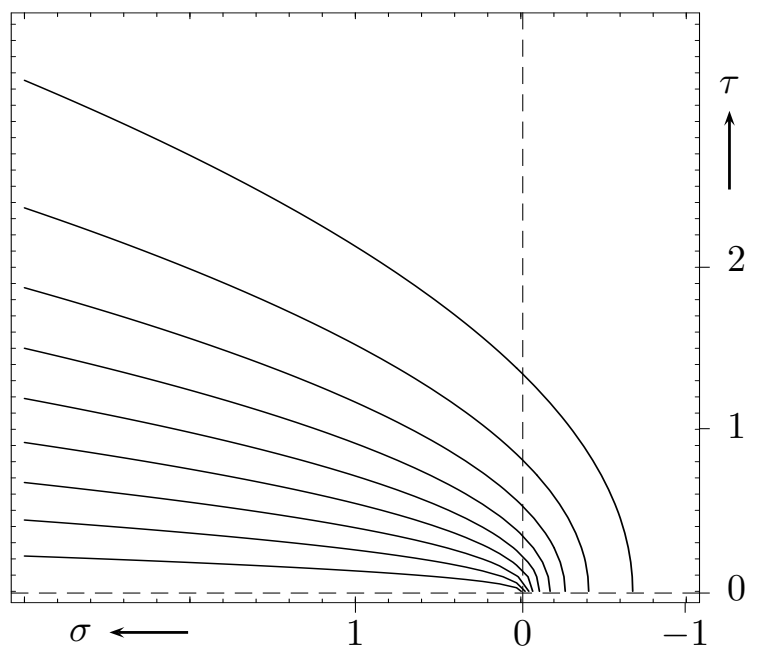

Figure 6: Isothermals for $T_{I I}$, based on $\bar{T}(\sigma, \tau)$ according to (93), in the boundary layer in the $(\sigma, \tau)$-plane near the flow front region

We have calculated a set of isothermals of $T_{I I}$ in the $(\sigma, \tau)$-plane inside the $G_{I I}$-boundary layer. The results are depicted in Figure 6. From this figure, we see that only a small part 
of the boundary layer enters the flow front region, $\sigma<0$. Moreover, we observe that the temperatures near the wall in the flow front region $G_{I I}$ are much higher (very close to 1) than those in the upstream direction $(\sigma \gg 1)$. This effect is caused by the convection due to the fountain flow in the front region. The same effect was found, by numerical simulations, by Kamal et al. [16, Fig. 10].

The boundary layer immediately behind the flow front, i.e. for $\sigma>0$, has to match with that in $G_{I I I}$; how this is done, will be shown in the next section.

\section{Temperature problem in the boundary layer in $G_{I I I}$}

\subsection{Differential equation and boundary conditions}

In Sections 3 and 4, we derived (stationary) solutions for the temperature in boundary layers near the upper and lower wall of the mould that are valid in $G_{I}$ and $G_{I I}$, respectively. We still need to find a solution for the temperature near the upper and lower walls in $G_{I I I}$, which couples the solution in $G_{I}$ to that in $G_{I I}$. Due to the symmetry of the problem, we only need to consider the solution near the upper wall, $Y=h$.

Written in dimensional coordinates, the temperature $T_{I I I}$ in $G_{I I I}$ satisfies the differential equation (8). We choose here the following scaling for $t, X, Y, U, V$, and $T$ :

- For $t, X, Y$, we take

$$
t=\frac{R}{V_{f}} \hat{t}, \quad X=R \hat{X}, \quad Y=h-\epsilon_{3} h \eta,
$$

where $0<\epsilon_{3} \ll 1$ but still unknown, and $\eta>0$ and of $O(1)$ with respect to $\epsilon_{3}$.

- Since already at distances a few times $h$ behind the flow front, the velocity resembles strongly that of a fully-developed flow, $V$ is taken equal to $V=0$ in $G_{I I I}$, and $U$ to

$$
U=\frac{3}{4} V_{f}\left(1-\left(\frac{Y}{h}\right)^{2}\right)=\frac{3}{4} V_{f} \epsilon_{3} \eta\left(2-\epsilon_{3} \eta\right) .
$$

- Similar to the previous sections, we scale $T$ according to

$$
T_{I I I}(\hat{X}, \eta, \hat{t})=\frac{T\left(R \hat{X}, h\left(1-\epsilon_{3} \eta\right), R \hat{t} / V_{f}\right)-T_{w}}{T_{i}-T_{w}} .
$$

Substitution of (107)-(108) into (8) renders the differential equation for the temperature $T_{I I I}$ in $G_{I I I}$ :

$$
\frac{\partial T_{I I I}}{\partial \hat{t}}+\frac{3}{4} \epsilon_{3} \eta\left(2-\epsilon_{3} \eta\right) \frac{\partial T_{I I I}}{\partial \hat{X}}=\frac{\lambda_{I I I}}{\epsilon_{3}^{2}}\left(\epsilon_{3}^{2} \delta^{2} \frac{\partial^{2} T_{I I I}}{\partial \hat{X}^{2}}+\frac{\partial^{2} T_{I I I}}{\partial \eta^{2}}\right)
$$

where

$$
\lambda_{I I I}:=\frac{\lambda R}{\rho c_{p} V_{f} h^{2}}=\delta \hat{\lambda}=O(\delta),
$$

see $(20)$.

Since the diffusion term in the $\hat{X}$-direction has a factor $\epsilon_{3}{ }^{2} \delta^{2}$, diffusion in the $\hat{X}$-direction may 
be neglected. In (109), we assume that the diffusion term in the $\eta$-direction is of the same order as the instationary term. Therefore, we take $\epsilon_{3}$ equal to $\epsilon_{3}=\sqrt{\lambda_{I I I}}=O\left(\delta^{1 / 2}\right) \ll 1$, which results in

$$
\frac{\partial T_{I I I}}{\partial \hat{t}}+\frac{3}{4} \epsilon_{3} \eta\left(2-\epsilon_{3} \eta\right) \frac{\partial T_{I I I}}{\partial \hat{X}}=\frac{\partial^{2} T_{I I I}}{\partial \eta^{2}} .
$$

The boundary conditions are given by

- For $\eta=0,0<\hat{X}<\hat{t}: T_{I I I}=0$.

- For $\eta \rightarrow 1 / \epsilon_{3}: T_{I I I} \rightarrow 1$.

- For $\hat{X} \rightarrow \hat{t}, 0<\eta<1 / \epsilon_{3}: T_{I I I} \sim T_{I I}$.

- For $\hat{X} \rightarrow 0,0<\eta<1 / \epsilon_{3}: T_{I I I} \sim T_{I}$.

\subsection{First-order solution}

We will approximate the solution of (111) for $T_{I I I}$ with the boundary conditions given above, by taking $\epsilon_{3} \rightarrow 0$ (hence $1 / \epsilon_{3} \rightarrow \infty$ ). This leads us to the differential equation for the first-order approximation $T_{I I I}^{1}$ of $T_{I I I}$ :

$$
\frac{\partial T_{I I I}^{1}}{\partial \hat{t}}=\frac{\partial^{2} T_{I I I}^{1}}{\partial \eta^{2}}
$$

with boundary conditions similar to the ones above.

Since (112) is independent of $\hat{X}$, we can solve the problem for each $\hat{X}$ separately. Note that (112) has a solution of the form

$$
T_{I I I}^{1}(\hat{X}, \eta, \hat{t})=C_{1}(\hat{X}) \operatorname{Erf}\left(\frac{\eta+C_{2}(\hat{X})}{2 \sqrt{\hat{t}+C_{3}(\hat{X})}}\right)+C_{4}(\hat{X}) .
$$

For $\eta=0$, we require for all $\hat{X}$ with $0<\hat{X}<\hat{t}$, that

$$
T_{I I I}^{1}(\hat{X}, 0, \hat{t})=C_{1}(\hat{X}) \operatorname{Erf}\left(\frac{C_{2}(\hat{X})}{2 \sqrt{\hat{t}+C_{3}(\hat{X})}}\right)+C_{4}(\hat{X})=0,
$$

yielding, $C_{2}(\hat{X})=C_{4}(\hat{X})=0$.

For $\eta \rightarrow \infty$, we require

$$
T_{I I I}^{1}(\hat{X}, \eta \rightarrow \infty, \hat{t})=C_{1}(\hat{X})=1,
$$

from which it follows that $C_{1}(\hat{X})=1$.

To determine $C_{3}(\hat{X})$, we must look at the behaviour of the temperature in region $G_{I I}$, where the temperature is given by (see (93)),

$$
T=\bar{T}(\sigma, \tau)=\operatorname{Erf}\left(\sqrt{\sqrt{\sigma^{2}+\tau^{2}}-\sigma}\right) .
$$


Here, $\sigma$ and $\tau$ are given by (49) and $\epsilon_{1}$ and $\epsilon_{2}$ by $\epsilon_{1}=\epsilon_{2}=2 \lambda_{I I}=2 \delta \lambda_{I I I}$ (see (56)). Hence, written in $\hat{X}$ and $\eta$ coordinates, first using (4) and then (106) and (??), $\sigma$ and $\tau$ are found to be equal to

$$
\sigma=-\frac{R}{h} \cdot \frac{x}{2 \epsilon_{3}{ }^{2}}=\frac{1}{2 \hat{\lambda}} \frac{\hat{t}-\hat{X}}{\delta^{3}}, \quad \tau=\frac{R}{h} \cdot \frac{1-y}{2 \epsilon_{3}{ }^{2}}=\frac{1}{2 \sqrt{\hat{\lambda}}} \frac{\eta}{\delta^{3 / 2}} .
$$

The temperature in $G_{I I}$, expressed in terms of $\hat{X}$ and $\eta$, follows from (116) as $(\hat{X}<\hat{t}$, and $\hat{X}$ not in a $\delta$-neighbourhood of $\hat{t}$ )

$$
\begin{aligned}
T_{I I}(\hat{X}, \eta, \hat{t}) & =\operatorname{Erf}\left(\sqrt{\frac{1}{2 \hat{\lambda} \delta^{3}}\left\{\sqrt{(\hat{t}-\hat{X})^{2}+\hat{\lambda} \delta^{3} \eta^{2}}-(\hat{t}-\hat{X})\right\}}\right) \\
& =\operatorname{Erf}\left(\frac{\eta}{2 \sqrt{\hat{t}-\hat{X}}}\left(1+O\left(\delta^{3}\right)\right)\right) .
\end{aligned}
$$

Comparing (118) with (113), we infer that $C_{3}(\hat{X})=-\hat{X}$. All this leads us to the following solution for $T_{I I I}^{1}$ :

$$
T_{I I I}^{1}(\hat{X}, \eta, \hat{t})=\operatorname{Erf}\left(\frac{\eta}{2 \sqrt{\hat{t}-\hat{X}}}\right)=\frac{2}{\sqrt{\pi}} \int_{0}^{\eta / 2 \sqrt{\hat{t}-\hat{X}}} e^{-s^{2}} d s .
$$

The first-order approximation of the temperature $T_{I}$ in $G_{I}$ is given by (37) as (with $X \rightarrow \hat{X}$ )

$$
T_{I}(\hat{X}, \zeta)=C \int_{0}^{\zeta / \sqrt[3]{\hat{X}}} e^{-\frac{2}{9} s^{3}} d s
$$

with $C$ given by (36).

We continue with the determination of the thickness of the boundary layer in $G_{I I I}$. Let $\eta_{b l}$ be the value of $\eta$ at the border of the boundary layer, where $T_{I I I}=1-\epsilon_{3}$. Using (119), we then find $\eta_{b l}$ from $\operatorname{Erfc}\left(\eta_{b l} / 2 \sqrt{\hat{t}-\hat{X}}\right)=\epsilon$, resulting in

$$
\frac{\eta_{b l}}{2 \sqrt{\hat{t}-\hat{X}}} \approx \sqrt{-\log \epsilon_{3}} \approx \sqrt{-\frac{1}{2} \log \delta},
$$

yielding for the thickness of the boundary layer

$$
\frac{h_{b l}}{h}=\sqrt{2 \hat{\lambda} \delta} \sqrt{\hat{t}-\hat{X}} \sqrt{-\log \delta}=\sqrt{2 \hat{\lambda}} \delta \sqrt{\frac{V_{f} t-X}{h}} \sqrt{-\log \delta} .
$$

The precise position of the interface between $G_{I I I}$ and $G_{I}$ is not so relevant for our further investigations; we are more interested in the part of $G_{I I I}$ in the vicinity of the flow front (directly behind the flow front). Therefore, a rather rough estimate for the transition zone between $G_{I I I}$ and $G_{I}$ suffices for us. With $\zeta=(3 / 4)^{1 / 3}(\hat{\lambda} \delta)^{1 / 6} \eta$, as follows from (23), (25), and (??), we see from (120) and (123) that $T_{I}$ and $T_{I I I}$ become of the same order when $\hat{X}$ becomes so small with respect to $\hat{t}$, that $\zeta / \sqrt[3]{\hat{X}}=(3 / 4 \hat{X})^{1 / 3}(\hat{\lambda} \delta)^{1 / 6} \eta \sim \eta / 2 \sqrt{\hat{t}-\hat{X}} \approx \eta / 2 \sqrt{\hat{t}}$, as is the case when $\hat{X} \sim 6(\hat{\lambda} \delta)^{1 / 2} \hat{t}^{3 / 2}$ (thus $\hat{X} / \hat{t}=O\left(\delta^{1 / 2}\right) \ll 1$, indeed). This indicates that the left boundary of $G_{I I I}$, i.e. the interface between $G_{I I I}$ and $G_{I}$, must be somewhere around $\hat{X} \sim 6(\hat{\lambda} \delta)^{1 / 2} \hat{t}^{3 / 2}$. 


\subsection{Second-order solution}

We can find a second-order solution of (111) by assuming that $T_{I I I}$ can be written in the form $T_{I I I}=T_{I I I}^{1}+\epsilon_{3} T_{I I I}^{2}+O\left(\epsilon_{3}^{2}\right)$, where $T_{I I I}^{2}$ is of order $O(1)$. Substituting this form into (111), and retaining only first order terms in $\epsilon_{3}$, we arrive at the differential equation for $T_{I I I}^{2}$ :

$$
-\frac{\partial T_{I I I}^{2}}{\partial \hat{t}}+\frac{\partial^{2} T_{I I I}^{2}}{\partial \eta^{2}}=\frac{3}{2} \eta \frac{\partial T_{I I I}^{1}}{\partial \hat{X}}=\frac{6}{\sqrt{\pi}} \frac{s^{3}}{\eta} e^{-s^{2}},
$$

according to (119), and with $s=\eta / 2 \sqrt{(\hat{t}-\hat{X})}$.

The boundary conditions for $T_{I I I}^{2}$ follow from the boundary conditions listed at the end of Section 5.1. However, the third and the fourth condition are only relevant for the matching of the three boundary layer solutions. Here, we discuss this matching only up to the first-order matching and, therefore, the latter two boundary conditions need not to be considered here. So, there remain:

- For $\eta=0,0<\hat{X}<\hat{t}: T_{I I I}^{2}=0$.

- For $\eta \rightarrow \infty: T_{I I I}^{2} \rightarrow 0$.

Equation (123) has a solution of the form $T_{I I I}^{2}(\hat{X}, \eta, \hat{t})=\eta \phi(s)$, where $\phi$ satisfies the differential equation:

$$
\frac{1}{2} s \phi^{\prime \prime}(s)+\left(1+s^{2}\right) \phi^{\prime}(s)=\frac{3}{\sqrt{\pi}} s^{2} e^{-s^{2}} .
$$

The general solution of (124) is given by

$$
\phi(s)=\frac{3}{8} \operatorname{Erf}(s)-\frac{3}{4 \sqrt{\pi}} s e^{-s^{2}}+C\left(\operatorname{Erf}(s)+\frac{1}{\sqrt{\pi} s} e^{-s^{2}}\right)+D .
$$

Hence, the general solution for $T_{I I I}^{2}$ in terms of $s$ and $\eta$ is given by

$$
T_{I I I}^{2}=\tilde{T}_{I I I}^{2}(s, \eta)=\eta\left(\frac{3}{8} \operatorname{Erf}(s)-\frac{3}{4 \sqrt{\pi}} s e^{-s^{2}}+C\left(\operatorname{Erf}(s)+\frac{1}{\sqrt{\pi} s} e^{-s^{2}}\right)+D\right),
$$

where $C$ and $D$ are to be determined by the two boundary conditions given above.

The first boundary condition says that $T_{I I I}^{2}=0$, for $\eta=0$, but then also $s=0$. Taking in (126) the limit for $\eta \downarrow 0$ and $s \downarrow 0$, we find that

$$
\tilde{T}_{I I I}^{2}(0,0)=\lim _{\eta \downarrow 0} \frac{C}{\sqrt{\pi}} \frac{\eta}{s}=\frac{C}{\sqrt{\pi}} \sqrt{\hat{t}-\hat{X}}=0,
$$

from which it follows that $C=0$.

For the second boundary condition, that is for $\eta \rightarrow \infty$, we have $s \rightarrow \infty$, and $T_{I I I}^{2} \rightarrow 0$. Since $\operatorname{Erf}(s) \rightarrow 1$ for $s \rightarrow \infty$, it must hold that $3 / 8+C+D=0$, and hence $D=-3 / 8$. Thus, we arrive at the second order solution for the temperature in $G_{I I I}$ :

$$
T_{I I I}^{2}=\tilde{T}_{I I I}^{2}(s, \eta)=\frac{3}{8} \eta\left(\operatorname{Erf}(s)-1-\frac{2}{\sqrt{\pi}} s e^{-s^{2}}\right) .
$$

In the next section, the effect of $T_{I I I}^{2}$ on the total solution is numerically evaluated; it is shown that the effect is small but observable. 


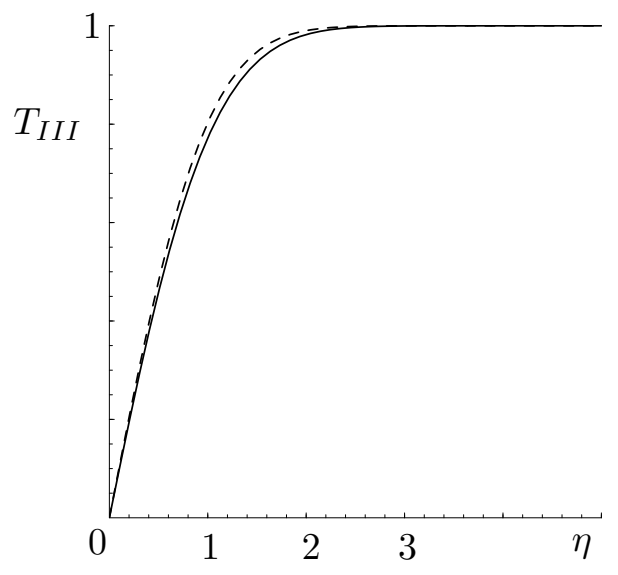

Figure 7: The first-order solution $T_{I I I}^{1}$ (dashed curve) and the second-order solution $T_{I I I}^{1}+$ $\epsilon_{3} T_{I I I}^{2}$ (solid curve) for the temperature in $G_{I I I}$ as a function of $\eta$ for $\hat{t}-\hat{X}=0.3$

\subsection{Numerical results and comparison of first- and second-order solutions}

We have calculated the temperature $T_{I I I}$ in some cross-section in $G_{I I I}$, i.e. at $\hat{t}-\hat{X}=0.3$, as a function of $\eta$, especially near the upper wall. We did this for both the first-order and the second-order solutions. The results are depicted in Figure 7. Comparing the two solutions, we conclude that the differences are small. We also did some numerical (FEM) computations and found corresponding results. From Figure 7, we observe that the temperature goes to its inlet value 1 for $\eta \approx 2$; for $\delta=0.01$, this means that the upper boundary occupies about $20 \%$ of the cross-section at $\hat{t}-\hat{X}=0.3$.

To illustrate the result of the matching of the inner solutions in the boundary layers of $G_{I I}$ and $G_{I I I}$ at the interface between these two regions, we have depicted in Figure 8 two isothermals, both for $\hat{T}=0.5$, in this interfacial region. This interface is closely behind the flow front region. In the left part of Figure 8 , we depict a global view in a $((\hat{t}-\hat{X}), \eta)$-plane on a length-scale of $(\delta) h$; this figure shows a perfect matching (in fact, the dashed curve and the solid curve almost completely overlap each other). To make the differences between $T_{I I}$ and $T_{I I I}$ more clear, we also draw a detailed view in a $(\sigma, \tau)$-plane on a length-scale of $\left(\delta^{2}\right) h$ in the right part of the figure (see (117) for the relationship between the respective length scales). Here, indeed some difference is observed (as it should be), but this figure also shows that these curves tend to overlap for increasing $\sigma$, as is confirmed by the left graph.

\section{NOTE}

Within a thin-layer approximation, often used for the simulation of injection moulding processes for narrow moulds, it is common practice to approximate the curved flow front $\Gamma$ by a straight line; see e.g. [2]. In this way, the influence of the shape of the flow front on the velocity and temperature distribution immediately behind the flow front is neglected. As it are especially these effects we are interested in, we did not follow this path here, but we will now try to indicate the inaccuracies in the near-by temperature caused by a straight-front approximation.

In for example [2], a thin-layer or lubrication-like approximation was used, yielding dominant 

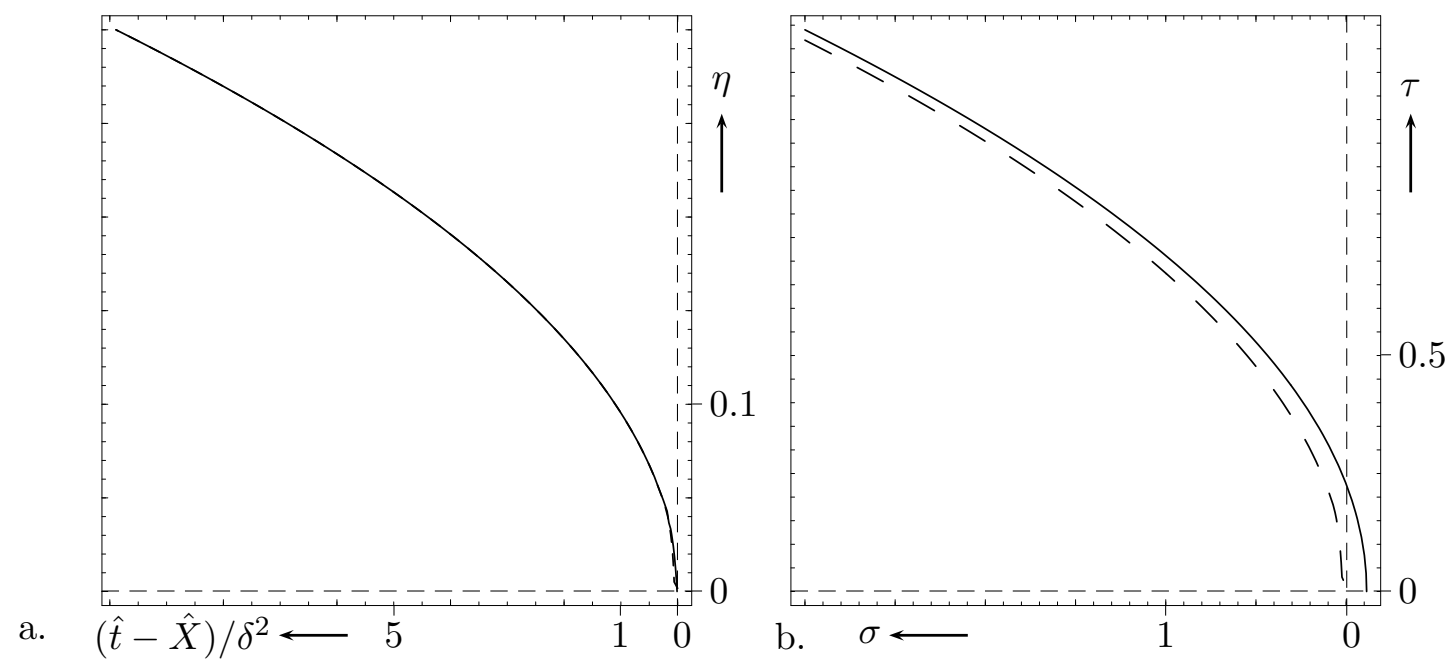

Figure 8: The isothermals $T_{I I}=0.5$ (solid curve) and $T_{I I I}=0.5$ (dashed curve), showing the matching of the boundary-layer temperatures $T_{I I}$ and $T_{I I I}$ near the flow front interface; a. global view in the $(\hat{t}-\hat{X}, \eta)$-plane (along the horizontal axis $(\hat{t}-\hat{X}) / \delta^{2}=(\hat{t}-\hat{X}) \times 10^{4}$ is plotted), b. detailed view in the $(\sigma, \tau)$-plane.

convection in the flow direction and dominant conduction in the thickness direction, whereby the heat conduction was assumed to prevail in the region closely behind the (straight) flow front. Neglecting heat convection in this region, they came to a heat diffusion equation (see [2, Eq. (25)]), exactly equal to our equation (112) for $T_{I I I}$. This means that in this approach the specific distribution, inclusive boundary layer, in $G_{I I}$ is completely discarded. The most typical effect there, namely that the temperature near the wall is much higher in the flow front region than in a point at the same distance of the wall in upstream direction, i.e. in $G_{I I}$, is then not found; see Section 4.3, or [16]. This is because this effect is due to the convection by the fountain flow in $G_{I I I}$, and it is precisely this convection that is neglected in the thin-layer approximation in e.g. [16]. It is evident that for an analysis of the temperature, and the associated viscous flow for a fluid of temperature-dependent viscosity, the above-mentioned effect is essential.

\section{Conclusions}

In the preceding sections, we have considered the heat flow in a fluid entering the narrow region between two fixed parallel plates. The fluid flow has a free surface, the flow front; the shape of the flow front (almost semi-circular) and the flow patterns in and behind the flow region (fountain flow and 2-D Poiseuille flow, respectively) have been calculated in a previous paper [1]. We found that in the bulk of the fluid the temperature remains (to first order) equal to the inlet temperature; this is due to the fact that the flow is convection dominated there. Only in boundary layers near the cooled walls the temperature drastically changes. We have found three different types of boundary layers referring to three distinct regions of the flow: $G_{I}, G_{I I}$ and $G_{I I I}$. We now review the results in these regions:

1. In $G_{I}$; the region far behind the flow front. 
In this region, the influence of the flow front can be neglected, and thus the flow here is a pure Poiseuille flow. The temperature in this region is stationary with respect to a fixed frame. The small parameter $\epsilon$, which is a measure for the thickness of the boundary layer, is here related to the dimensionless heat conduction coefficient $\lambda_{I}=4 \hat{\lambda} \delta / 3=O(\delta) \ll 1$, according to $\epsilon=\sqrt[3]{4 \hat{\lambda} / 3} \delta^{1 / 3}$. The inner solution within the boundary layer is governed by a convection-diffusion equation for the temperature $T$, whereas the outer solution, outside the boundary layer, i.e. in the kernel of the flow, is convection dominated. The inner solution was found in terms of a similarity solution, and both a first order $(O(\epsilon))$ and a second order $\left(O\left(\epsilon^{2}\right)\right)$ asymptotic solution was derived. Despite the fact that the parameter $\epsilon$ was not really very small $(\epsilon=0.21)$ the differences between the first- and second- order solutions were minimal (only of some relevance very near the walls). The asymptotic solution was compared with a numerical solution, and again the agreement was very good. That, for $G_{I}, \epsilon$ is not very small implies that the boundary layer in this region is relatively thick. If we define the border of the boundary layer as the place where the scaled temperature differs $O(\epsilon)$ from the scaled inlet temperature, we found for the thickness $h_{b l}$ of the boundary layer in $G_{I}$

$$
\frac{h_{b l}}{h}=\sqrt[3]{-2 \hat{\lambda} \delta \hat{X} \log \delta}
$$

In Section 5.2, we derived that the values for $X$ in the region $G_{I}$ are restricted by $0<\hat{X}<6(\hat{\lambda} \delta)^{1 / 2}\left(V_{f} t / R\right)^{3 / 2}$. Hence, the region $G_{I}$ is time dependent, and its area is increasing with time.

2. In $G_{I I}$; the flow front region.

The type of flow in this region is a fountain flow, which is stationary with respect to a comoving frame (moving with the constant speed of propagation of the flow front). Therefore, also the temperature in $G_{I I}$ is taken to be stationary with respect to the comoving frame. In the main part of $G_{I I}$ the heat flow is convection dominated, implying that $T=T_{i}$ in $G_{I I}$, except for two small boundary layers in the upper and lower tips of the flow front region. These layers extend to narrow layers at the walls in the region immediately behind the flow front. The small parameter $\epsilon$ here is $\epsilon=\lambda_{I I}=2 \delta^{2} \hat{\lambda}$, which is much smaller than the one in $G_{I}$, so the boundary layers here are much thinner than those in $G_{I}$. The inner solution, of convection-diffusion type, was found by means of a Wiener-Hopf technique. It turned out that this solution could be written as a similarity solution, i.e. as $T_{I I}(\sigma, \tau)=\operatorname{Erf}(\rho)$, where $\rho=\rho(\sigma, \tau)=\sqrt{\sqrt{\sigma^{2}+\tau^{2}}-\sigma}$. We found that the thickness $h_{b l}$ of the boundary layer as function of $\sigma$ is given by

$$
\frac{h_{b l}}{h}=2 \hat{\lambda} \delta^{2} \rho_{b l}^{2} \sqrt{1+\frac{2 \sigma}{\rho_{b l}^{2}}} \approx-\hat{\lambda} \delta^{2} \log \delta \sqrt{1-\frac{4 \sigma}{\log \delta}} .
$$

Moreover, the right end point of this layer, where $h_{b l} \downarrow 0$ was found for $\sigma \rightarrow \log \delta$.

3. In $G_{I I I}$; the intermediate region between $G_{I}$ and $G_{I I}$.

Also in the intermediate region $G_{I I I}$ a boundary layer exists, forming the connection between the boundary layers in $G_{I}$ and $G_{I I}$. The small $\epsilon$-parameter here is 
$\sqrt{\lambda_{I I}}=O\left(\delta^{1 / 2}\right)$, which is large compared to the $\epsilon$-parameter in $G_{I I}$ and small compared to the one in $G_{I}$. Again, an inner solution was found as a similarity solution; see (119). Moreover, a second-order solution was derived, and the results of the first- and second-order solutions were compared to each other, and to a numerical solution; good agreement was found.

In Section 5.2, we showed that the solution in $G_{I I I}$ matches perfectly with the inner solution in $G_{I I}$, while a good matching with the solution in $G_{I}$ is obtained when the interface between $G_{I I I}$ and $G_{I}$ is taken somewhere near to $\hat{X}=(\hat{\lambda} \delta)^{1 / 2}\left(V_{f} t / R\right)^{3 / 2}$. Hence the region $G_{I I I}$ ranges from $\hat{X}=(\hat{\lambda} \delta)^{1 / 2}\left(V_{f} t / R\right)^{3 / 2}$ to $\hat{X} \approx V_{f} t / R$. Therefore, in the region which we refer to as "region (closely) behind the flow front" we must use for the temperature the solution found for $G_{I I I}$.

In Section 5.2, we have found for the thickness of the boundary layer

$$
\frac{h_{b l}}{h}=\sqrt{2 \hat{\lambda}} \delta \sqrt{\frac{V_{f} t-X}{h}} \sqrt{-\log \delta},
$$

which is of the same order as the one found in (105) (the difference of the factor 2 is due to the two different $\epsilon$-parameters used in $G_{I I}$ and $\left.G_{I I I}\right)$. Hence, we can conclude that the boundary layers from $G_{I I}$ and $G_{I I I}$ match perfectly.

From (129) and (131), we see that the borders of the boundary layers in $G_{I}$ and $G_{I I I}$ coincide at (provided $\hat{X} \ll \hat{t}$ )

$$
\hat{X}=\frac{4 \sqrt{2}}{3}(-\hat{\lambda} \delta \log \delta)^{1 / 2}(\hat{t})^{3 / 2} .
$$

Note that this place is within the range of the interface between $G_{I}$ and $G_{I I I}$ as estimated at the end of Section 5.2 by matching.

To conclude, we like to explain in how far, in our view, the results obtained here can be used for an investigation of flow front instabilities. We found that the temperature distribution shows boundary layers near the walls of the mould. The hope that these results can be used for an explanation of the instability (wobbling) of the flow front is based on the expectation that the wobbling could be driven by asymmetric temperature distributions. This asymmetry influences the flow, and this is most manifest in the boundary layers. In this context, we notice that an asymmetric flow front causes differences in temperature, and thus also in viscosity.

To explain our view further, let us assume there is initially an asymmetric flow front (for instance due to an asymmetric inlet of the mould) and that at certain time $t$ the contact point of the flow front with the upper wall is farther (in the $X$-direction) than the corresponding point at the lower wall, i.e. $\xi_{f}(+h, t)>\xi_{f}(-h, t)$. In that case, the fluid near the upper wall will cool more than the fluid near the lower wall, and so the fluid will become stiffer (larger viscosity) at the upper wall than at the lower one. Consequently, the fluid will be retarded at the upper wall with respect to the fluid at the lower wall and, thus, the asymmetry will flip. This means that at a somewhat later time the flow front is farther at $Y=-h$ than at $Y=+h$. This cyclus will repeat itself and then the front is wobbling. Hence, the differences in viscosity can induce an ongoing asymmetry of the flow front, which can result into instability. 
Acknowledgement The authors thank Stef van Eijndhoven and Joop Boersma for stimulating discussions and valuable suggestions for improvements on the manuscript.

\section{References}

[1] H.J.J. Gramberg, J.C.W. van Vroonhoven, and A.A.F. van de Ven, Flow patterns behind the free flow front for a Newtonian fluid injected between two infinite parallel plates, European Journal of Mechanics-B/Fluids 23 (2004) In press.

[2] H. Mavridis, A.N. Hrymak and J. Vlachopoulos, The Effect of Fountain Flow on Molecular Orientation in Injection Moulding, Journal of Rheology 32 (1988) 639-663.

[3] D.P. Wall and S.K. Wilson, The linear stability of channel flow of fluid with temperaturedependent viscosity, Journal of Fluid Mechanics 323 (1996) 107-132.

[4] H.A. Attia, Transient MHD flow and heat transfer between two parallel plates with temperature dependent viscosity, Mechanics Research Communications 26 (1999) 115121.

[5] A. Pinarbasy and C. Ozalp, Effect of viscosity models on the stability of a Non-Newtonian fluid in a channel with heat transfer, Int. Comm. Heat Mass Transfer 28 (2001) 369-378.

[6] D.J. Estep, S.M. Verduyn Lunel, and R.D. Williams, Analysis of shear layers in a fluid with temperature-dependent viscosity, Journal of Computational Physics 173 (2001) 1760 .

[7] L.E. Becker and G.H. $\mathrm{M}^{c}$ Kinley, The stability of viscoelastic creeping plane shear flows with viscous heating, J. Non-Newtonian Fluid Mech. 92 (2000) 109-133.

[8] Nguyen-Chung, T., and G. Mennig, Non-isothermal transient flow and Molecular orientation during injection mold filling, Rheologica Acta 40 (2001) 67-73.

[9] H.J.J. Gramberg and A.A.F. van de Ven, Thermally induced flow front instabilities in injection moulding. In: Progress in Industrial Mathematics at ECMI 2000. Ed. A.M. Anile, V. Capasso, A. Greco, Springer: Berlin (2002) 433-438.

[10] A.A.F. van de Ven, Modelling of industrial processes for polymer melts; Extrusion and injection moulding. In: Mathematical Modelling for Polymer Processing. Polymerization, Cristallization, Manufacture. Ed. Capasso, Springer: Berlin (2002) 263-309.

[11] H.J.J. Gramberg, Thesis, forthcoming: (2003) .

[12] P.Kennedy, Flow analysis in injection molds, Munich: Hanser Publishers (1995) .

[13] B. Noble, Methods Based on the Wiener-Hopf Technique, London: Pergamon Press (1958) . 
[14] G.F. Carrier, M. Krook and C.E. Pearson, Functions of a complex variable, theory and technique, New York: $\mathrm{M}^{c}$ Graw-Hill book company (1966) .

[15] Fritz Oberhettinger, Tabellen zur Fourier Transformation, Berlin: Springer (1957) .

[16] Kamal, M.R., Goyal, S.K., and E. Chu. Simulation of injection mold filling of viscoelastic polymer with fountain flow, AIChE Journal, 341988 (94-106) 